ARTICLE

DOI: $10.1038 /$ s41467-018-06944-1

\title{
Candida albicans gains azole resistance by altering sphingolipid composition
}

\author{
Jiaxin Gao ${ }^{1,2,3}$, Haitao Wang ${ }^{4,5}$, Zeyao Li, ${ }^{1,6}$, Ada Hang-Heng Wong (1) 4, Yi-Zheng Wang ${ }^{1}$, Yahui Guo ${ }^{7}$, Xin Lin ${ }^{7}$, \\ Guisheng Zeng ${ }^{5}$, Haoping Liu $^{3}$, Yue Wang (1) ${ }^{5,8}$ \& Jianbin Wang (1) 1,2
}

Fungal infections by drug-resistant Candida albicans pose a global public health threat. However, the pathogen's diploid genome greatly hinders genome-wide investigations of resistance mechanisms. Here, we develop an efficient piggyBac transposon-mediated mutagenesis system using stable haploid C. albicans to conduct genome-wide genetic screens. We find that null mutants in either gene FEN1 or FEN12 (encoding enzymes for the synthesis of very-long-chain fatty acids as precursors of sphingolipids) exhibit resistance to fluconazole, a first-line antifungal drug. Mass-spectrometry analyses demonstrate changes in cellular sphingolipid composition in both mutants, including substantially increased levels of several mannosylinositolphosphoceramides with shorter fatty-acid chains. Treatment with fluconazole induces similar changes in wild-type cells, suggesting a natural response mechanism. Furthermore, the resistance relies on a robust upregulation of sphingolipid biosynthesis genes. Our results shed light into the mechanisms underlying azole resistance, and the new transposon-mediated mutagenesis system should facilitate future genome-wide studies of C. albicans.

\footnotetext{
${ }^{1}$ School of Life Sciences, Tsinghua University, Beijing 100084, China. ${ }^{2}$ Centre for Life Sciences, Tsinghua University, Beijing 100084, China. ${ }^{3}$ Department of Biological Chemistry, University of California, Irvine, CA 92697, USA. ${ }^{4}$ Faculty of Health Sciences, University of Macau, Macau, China. ${ }^{5}$ Institute of Molecular and Cell Biology, Agency for Science, Technology and Research, Singapore 138673, Singapore. ${ }^{6}$ Peking-Tsinghua-NIBS Joint Graduate Program, Tsinghua University, Beijing 100084, China. ${ }^{7}$ Institute for Immunology, School of Medicine, Tsinghua University, Beijing 100084, China. ${ }^{8}$ Department of Biochemistry, Yong Loo Lin School of Medicine, National University of Singapore, Singapore 117596, Singapore. Correspondence and requests for materials should be addressed to Y.W. (email: mcbwangy@imcb.a-star.edu.sg) or to J.W. (email: jianbinwang@tsinghua.edu.cn)
} 
C andida albicans is a major fungal pathogen of humans, affecting millions of people and kills hundreds of thousands worldwide annually ${ }^{1}$. Mortality rates of invasive $C$. albicans infection remain high despite the treatment with existing antifungal therapies. Multidrug-resistant Candida species and strains are rapidly emerging and spreading globally, threatening to render our entire antifungal arsenal obsolete ${ }^{2}$. Azoles are common first-line drugs against most Candida species in systematic infections, which act by inhibiting the 14a-demethylase Erg11 in the ergosterol biosynthesis pathway and causing the accumulation of the toxic sterol 14,24-dimethylcholesta-8,24(28)dien-3 $\beta, 6 \alpha$-diol (DMCDD) that permeabilizes the plasma membrane ${ }^{3}$. Extensive research has demonstrated that fungal pathogens can quickly evolve various resistance strategies after exposure to an antifungal agent. C. albicans can gain resistance to azoles via genetic alteration of the drug target Erg11, upregulation of efflux pumps Cdr1, Cdr2, and Mdr1, and inactivation of Erg3 that synthesizes the toxic sterol ${ }^{4,5}$. However, our mechanistic understanding of antifungal resistance is incomplete ${ }^{2}$. It remains unclear why some fungal species are intrinsically resistant or easily acquire resistance to multiple antifungal drugs. Thus, it is imperative to identify and understand the molecular mechanisms that govern antifungal resistance.

Genome-wide genetic screening is a powerful tool to identify genes involved in a biological process. However, it has been difficult to apply these technologies to C. albicans due to its diploid genome. Although several recent studies conducting large-scale genetic screens of $C$. albicans mutant libraries resulted in important findings ${ }^{6-10}$, investigation of many aspects of C. albicans biology still relies on inference from data obtained in the studies of Saccharomyces cerevisia ${ }^{11,12}$. This approach inevitably misses out genes that perform specific functions and determine unique traits in C. albicans. For example, S. cerevisiae is nonpathogenic and does not grow true hyphae which are the predominant morphology of $C$. albicans in host tissues and a key virulence factor ${ }^{11}$. Currently, most C. albicans genes with limited homology to $S$. cerevisiae remains uncharacterized ${ }^{13}$.

Recently, some haploid C. albicans strains were isolated ${ }^{14}$. Although avirulent, the haploids possess most traits that define the species including the ability to undergo the yeast-hyphae transition, the white-opaque phenotypic switch, the formation of chlamydospores, and mating ${ }^{14}$. The haploids have opened up opportunities for scientists to explore large-scale, genome-wide genetic screening strategies in this pathogen. The recent application of CRISPR-cas9 technology in haploid C. albicans in the identification of new virulence regulators and genetic networks demonstrates the massive potential of using haploid C. albicans in genetic screens ${ }^{15}$.

Transposon-mediated mutagenesis is a widely used genetic tool. Transposons are movable genetic units and have two essential components, a transposase and a pair of transposonspecific inverted terminal repeats (ITRs). One example is the piggyBac $(P B)$ transposable element, first isolated from the moth Trichoplusia $n i$ and later adapted to other organisms from bacteria to mammals ${ }^{16,17} . P B$ transposase recognizes the ITRs and moves the entire content from one chromosomal locus and integrates it at a TTAA motif in a new locus ${ }^{18} . P B$ transposition occurs via a precise cut-and-paste mechanism which leaves no footprints behind, resulting in a full restoration of the donor site $^{19}$. Thus, a phenotype caused by a $P B$ insertion can be easily verified by phenotype reversion. Furthermore, $P B$ transposon inserts randomly throughout the genome $\mathrm{e}^{20}$.

In this study, we develop a highly efficient, $P B$-based mutagenesis system using stable haploid $C$. albicans strains. We apply this technology to conduct genome-wide genetic screens for genes involved in resistance to the antifungal drug fluconazole and discover a new resistance mechanism.

\section{Results}

Construction of the piggyBac transposition system in C. albicans. We first optimized the piggyBac transposase (PBase) gene for use in C. albicans. We replaced all CUG codons with UUG because CUG encodes serine instead of the conventional leucine in C. albicans, added a $3 \times \mathrm{SV} 40$ nuclear localization signal to its $\mathrm{N}$-terminus, and controlled the expression using the tetracyclineinducible (Tet-On) promoter (Fig. 1a). Also, we mutated seven amino-acid residues to generate a hyperactive transposase according to previous studies ${ }^{21}$. We then integrated this CaPBase gene, using SAT1 as the selectable marker, at the $A D H 1$ locus of the stable, uridine auxotrophic (Ura-) haploid C. albicans strain GZY803 22 to generate the YW01 CaPBase-expressing strain.

To test whether $P B$ transposition would occur in $C$. albicans, we transformed the donor plasmid pPB[URA3] carrying the ITRURA3-ITR cassette into YW01 cells that had been grown in the absence or presence of doxycycline (Dox) for $48 \mathrm{~h}$ and then spread the cells onto uridine-deficient (-Uri) plates. The transformation of Dox-induced cells led to the growth of numerous colonies, while the uninduced cells produced only a few likely due to the basal expression of CaPBase (Fig. 1b), an event that happened in $<0.001 \%$ of the cells. This result indicated that the Dox-induced CaPBase excised the $P B$ cassette from the plasmid and integrated it into the genome.

Previous studies in other organisms showed that $P B$ excision did not leave any footprint behind ${ }^{19}$. To confirm this characteristic in C. albicans, we constructed a C. albicans strain in which we inserted the PB[URA3] cassette at a TTAA site (n.t. 709-712) within the open reading frame (ORF) of $A R G 4$ (Fig. 1c), yielding YW02 that is arginine auxotrophic (Arg-) and uridine prototrophic $(\mathrm{Ura}+)$ and also expresses CaPBase from the Tet-On promoter at the $A D H 1$ locus. Precise excision of $P B$ would convert YW02 cells from Arg- to Arg+. After Dox induction for $48 \mathrm{~h}$, we spread the YW02 cells onto glucose minimal medium (GMM) plates, picked 20 independent colonies, and confirmed that all 20 clones grew well on -Arg-Uri plates (Fig. 1d), indicating that the cells had excised PB[URA3] out of $A R G 4$ precisely. This was confirmed by site-specific PCR using two pairs of primers as shown in Fig. 1c (Fig. 1e shows examples of 5 clones) and DNA sequencing analysis of the PCR products. To map $P B$ insertion sites, we performed inverse PCR as described in Fig. If which allowed amplification of the DNA fragment encompassing a transposon junction. DNA sequencing analysis of the PCR products and homology search of the Candida Genome Database (CGD) located the $P B$ insertion sites on all eight chromosomes (Supplementary Table 1), seven within ORFs and 13 in intergenic regions (Fig. 1g). Our results demonstrated random $P B$ transposon insertion and precise excision in $C$. albicans.

Ploidy and $P B$ transposition efficiency analysis. Autodiploidization observed in C. albicans haploids has been a major concern regarding their applicability in genetic screens ${ }^{14}$. To evaluate ploidy stability during transposition, we first performed qPCR analysis to measure CaPBase expression levels at timed intervals in YW02 cells grown on solid media with or without Dox. The results demonstrated a sharp increase of CaPBase expression upon Dox addition (Fig. 2a). In parallel, we examined cell ploidy by flow cytometry and found that the cells retained $1 \mathrm{~N}$ DNA content for at least $72 \mathrm{~h}$ (Fig. 2b) regardless of Dox addition, indicating a stable ploidy during the robust expression of 
a

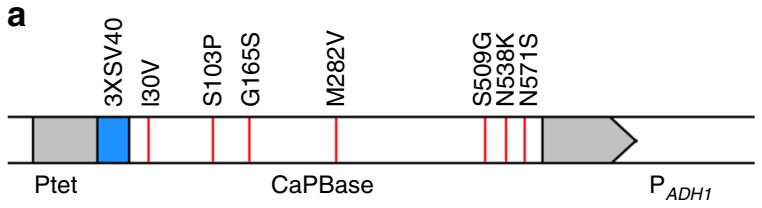

C

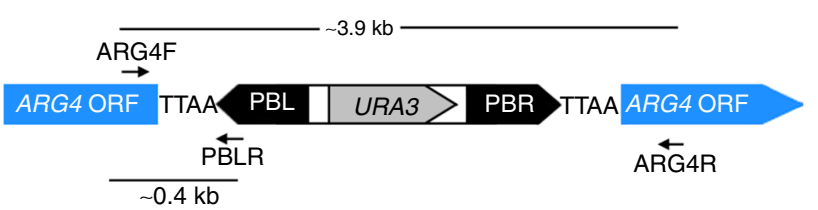

d

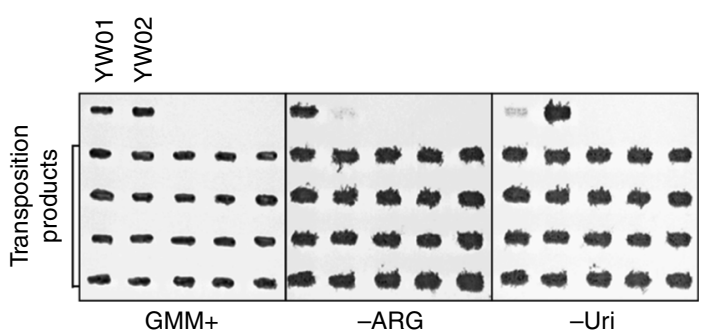

e

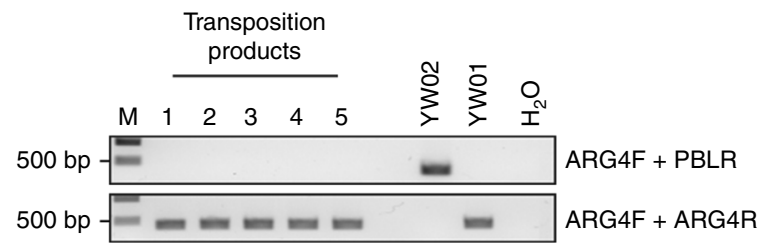

b

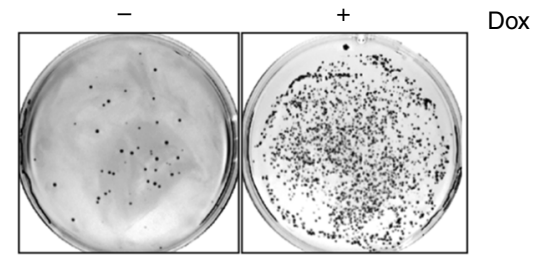

f

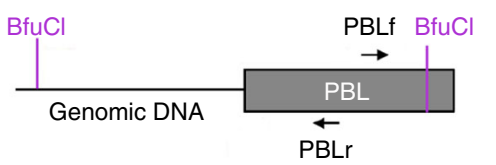

Digestion with BfuC

Self-ligation

g
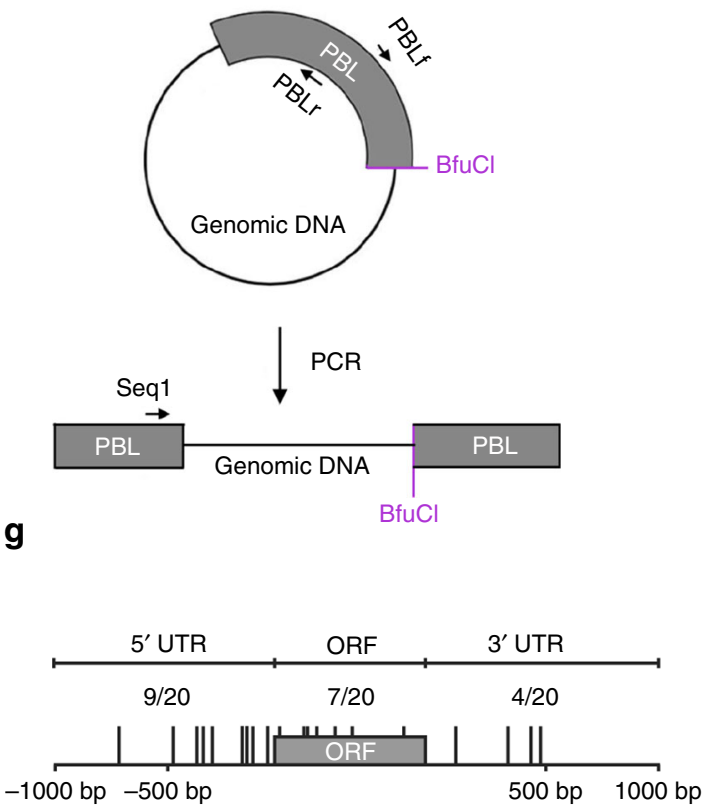

Fig. 1 PB transposition in haploid C. albicans. a Schematic description of the CaPBase construct inserted at the ADH1 locus. Ptet, Tet-On promoter; $3 X S V 40$, SV40 nuclear localization signal; and $\mathrm{P}_{A D H 1}, A D H 1$ promoter. Hyperactive mutations are indicated along the top. $\mathbf{b}$ Selection of transformants with $\mathrm{PB}$ [URA3] integration on plates. YW01 cells were grown in YPD for $48 \mathrm{~h}$ in the presence $(+)$ or absence ( - ) of Dox before transformation with pPB[URA3]. Transformation products were spread onto GMM plates ( $90 \mathrm{~mm}$ Petri dishes). c Schematic description of the PB[URA3] cassette integrated within ARG4. Arrows indicate the PCR primers used to detect $P B$ excision. d Confirmation of the auxotrophic phenotypes of transposition products of YW02. Strains grown on a GMM+Uri+Arg+His (GMM+) plate were replica-transferred onto a GMM+Uri+His (-Arg) or GMM+Arg+His (-Uri) plate and then incubated at $30^{\circ} \mathrm{C}$ overnight. YWO1 and YW02 were included as controls. e Precise excision of $P B$ from the ARG4 locus. Representative gel electrophoresis results of PCR products amplified from YW02 transposition products using primers shown in $\mathbf{c}$. $\mathbf{f}$ Schematic description of the identification of $P B$ insertion sites by inverse PCR. Arrows indicate the PCR (PBLf and PBLr) and sequencing (Seq1) primers used. $\mathbf{g}$ Distribution of PB insertion sites in and around ORFs in YW02 transposition products. Positions in the ORF were determined by treating the entire length of an ORF as 100 and then determining the relative position of an insertion in the ORF. Positions in the $5^{\prime}$ or $3^{\prime}$ region were determined by whether the insertion was closest to the $5^{\prime}$ or $3^{\prime}$ end of an ORF

CaPBase. Tests in liquid medium produced similar results (Supplementary Figure 1a and $1 \mathrm{~b}$ ).

We next estimated $P B$ transposition frequency. Aliquots of YW02 cells grown on solid medium containing Dox were harvested at intervals, and each was divided into two equal parts, and one was spread onto a YPD plate that allows all cells to grow and the other onto GMM+histidine (His) plates where only Arg + Ura + transposants can grow. Transposition frequency was calculated by dividing the number of colonies on the GMM plate with that on the YPD plate. Figure $2 \mathrm{c}$ shows that $P B$ transposition frequency increased with time during Dox induction, reaching $\sim 5 \%$ at $72 \mathrm{~h}$. We also achieved a similar frequency in liquid medium (Supplementary Figure 1c).

Multiple transposon insertions in a genome complicate subsequent identification of the mutation responsible for a phenotype. Although a single copy of $P B$ transposon exists at the $A R G 4$ locus in YW02, the copy number may increase when transposition happens in $\mathrm{S}$ or $\mathrm{G} 2$ cells $^{23}$. To test whether $P B$ copy number changes during transposition, we analyzed the copy number of PB[URA3] in transposants. After $24 \mathrm{~h}$ of CaPBase induction, we spread the culture onto GMM+His plates to select transposants and randomly picked 10 colonies. We performed 
a

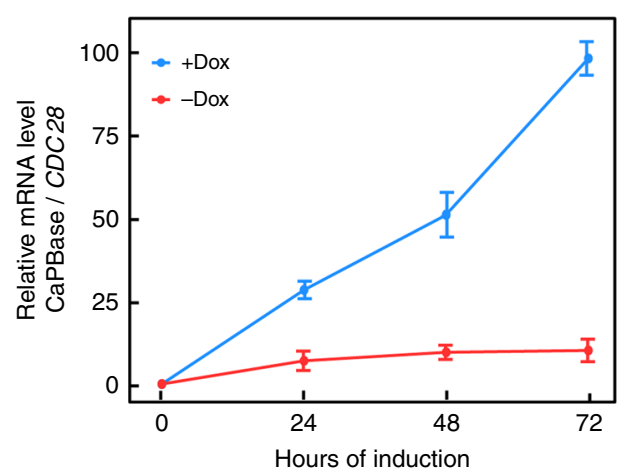

C

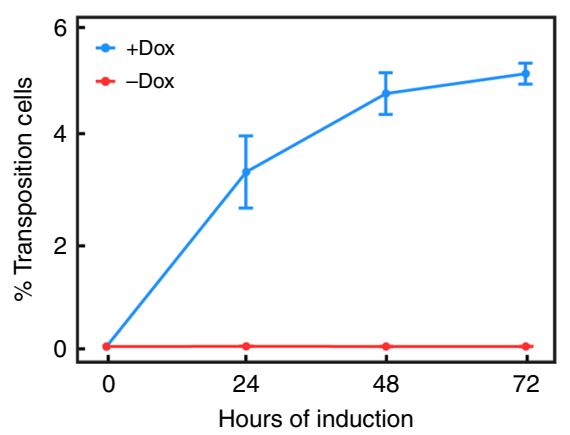

b

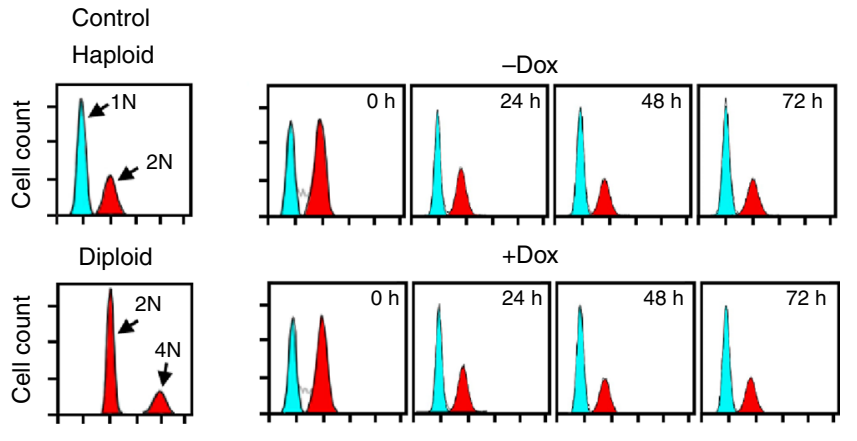

d

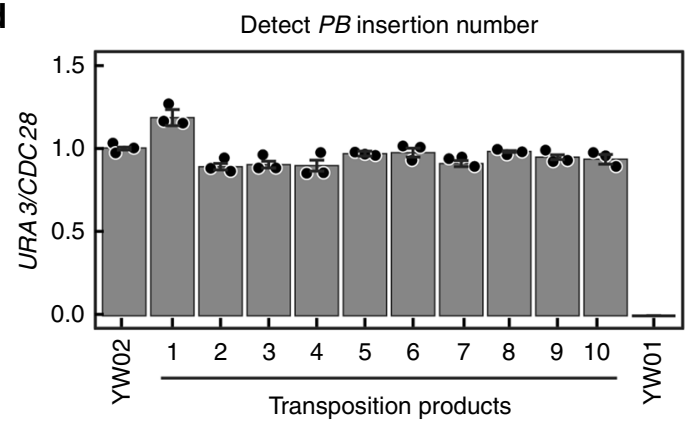

e
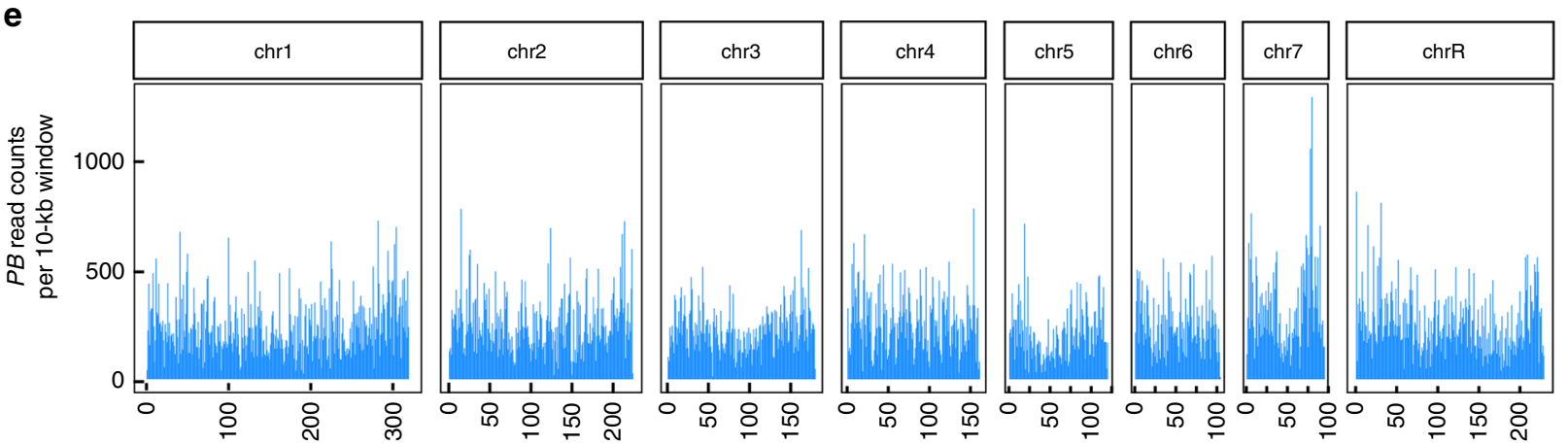

$\mathbf{f}$
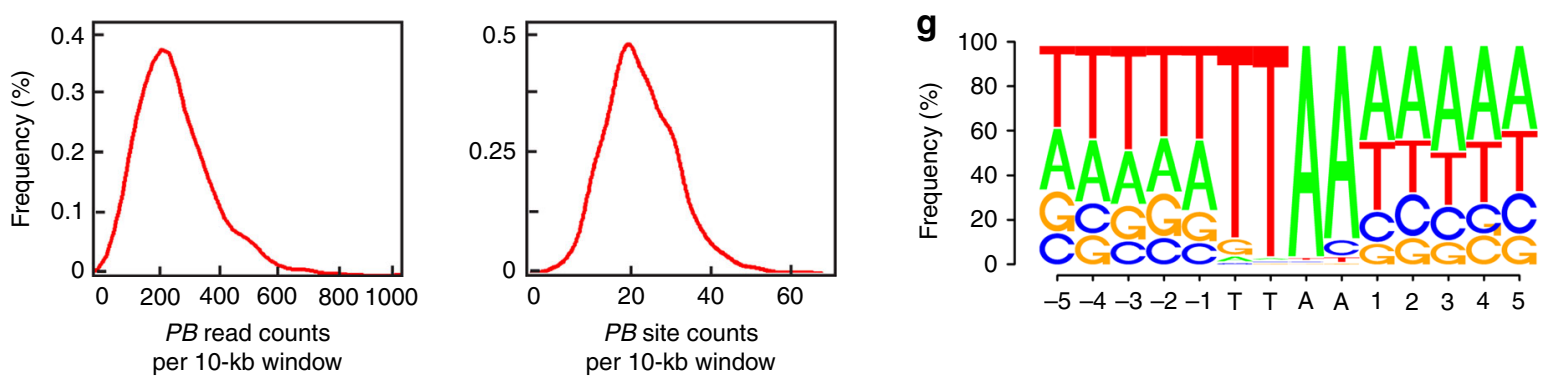

Fig. 2 PB transposition and insert distribution in C. albicans genome. a qPCR analysis of CaPBase expression during transposition induction. CDC28 mRNA level was used for normalization. Error bars represent standard deviation (s.d.) from the mean of triplicate samples. b Ploidy analysis during transposition induction by flow cytometry. The DNA content of induced cells at each time point was compared with that of haploid and diploid control strains. c Estimation of transposition efficiency in YW02. See the text for description. Error bars represent s.d. from the mean of three independent experiments. d Analysis of $P B$ copy number in transposition products by comparing the levels of URA3 and CDC28 DNA using qPCR. YW01 and YW02 were included as controls. Error bars represent s.d. from the mean of triplicate samples. e Genome-wide NGS analysis of $P B$ distribution. $P B$-specific reads were plotted in 10 $\mathrm{kb}$ sliding windows. f Histogram of $P B$-specific read counts and $P B$-specific site counts per 10-kb window. $\mathbf{g}$ Motif analysis of $P B$-insertion sites

qPCR to determine the level of $P B$ DNA in comparison with a control gene $C D C 28$ in the genome. All clones were found to contain a single copy of $P B$ (Fig. $2 \mathrm{~d}$ and Supplementary Figure 1d), indicating that the vast majority of transposants harbored a single $P B$ insertion in the genome.
Genome-wide analysis of $\boldsymbol{P B}$ transposon distribution. Next, we analyzed $P B$ distribution in the genome using next-generation sequencing (NGS). Transposition was induced with Dox for $24 \mathrm{~h}$, and then Arg+Ura+ transposants were selected on GMM+His plates. Genomic DNA was extracted from the transposant pool 
and subjected to NGS. We acquired 11,595,368 overlapping read pairs, each containing one part matching the $P B$ element and the other unambiguously matching a sequence of the $C$. albicans genome. The $P B$ transposon distribution in $10 \mathrm{~kb}$ non-overlapping windows is largely uniform throughout the genome (Fig. 2e and Supplementary Figure 1e). On average, there were 20 unique insertion sites in a 10-kb window (Fig. $2 \mathrm{f}$ ), i.e., $\sim 1$ every $500 \mathrm{bp}$. We detected $P B$ inserts in nearly 5000 genes out of a total of 6466 annotated and predicted genes in CGD. The failure to find $P B$ insertion in the rest of the genes is likely due to their essentiality or the fitness cost caused by the $P B$ insertion, consistent with the $\sim 1100$ essential genes in S. cerevisiae ${ }^{24}$.

Nucleotide motif analysis of the insertion sites revealed $82.4 \%$ at TTAA sites, $4.5 \%$ at TTAG, $4.4 \%$ at CTAA, $3.6 \%$ at TTGT, and $5.1 \%$ at other non-specific sites (Fig. 2g). Also, we found significant enrichment of $\mathrm{Ts}$ and As in the 5 nucleotides immediately upstream and downstream of the $P B$ inserts, respectively (Fig. $2 \mathrm{~g}$ ). $P B$ insertion occurred more frequently in promoter regions than in ORFs. This could be due to the enrichment of TA dinucleotides in promoters, which facilitates the unwinding of double-stranded DNA during transcription initiation. Another reason could be the fitness cost caused $P B$ insertion in some ORFs, which may reduce their presence in the transposant population.

$P B$ mutagenesis for forward genetic screens of 5-FOA resistant mutants. To evaluate the effectiveness of the $P B$ transposon system in genetic screens in C. albicans, we performed a proof-ofprinciple screen for mutants resistant to 5-fluoroorotic acid (5FOA). As 5-FOA is converted to the toxic 5-fluorouracil in strains expressing a functional $U R A 3^{25}$, we wanted to determine how frequently we can identify ura3 mutants in the transposon insertion library. We constructed a starting strain YW05 by using URA3 to delete HIS1 in GYZ803 and then inserting a PB[HIS1] cassette in ARG4 ORF. YW05 also expresses CaPBase from the $A D H 1$ locus. Figure 3a describes the steps of the screen. YW05 cells were grown as patches on YPD plates containing Dox for 48 $\mathrm{h}$ to induce the transposition. Then, the cells were replicatransferred to GMM+Uri plates to enrich Arg+His+ transposants which were then transferred to GMM+Uri+5-FOA plates to select for 5-FOA-resistant mutants. Among 30 colonies randomly picked from the 5-FOA plates were 26 true 5-FOAresistant mutants (Fig. 3b) and 4 clones (\#3, \#7, \#18, and \#21) were false and thus excluded from further characterization. Colony PCR using a pair of primers flanking URA3 failed to detect the 1.4-kb amplicon expected from the WT strain in 22 of the 26 5-FOA-resistant mutants (Fig. 3c), suggesting $\mathrm{PB}$ [HIS1] insertion in URA3. URA3 seemed intact in four clones, \#4, \#9, \#15, and \#26, suggesting $P B$ disruption of other genes leading to 5-FOA resistance (Fig. 3c). Next, we performed PCR analysis of the 22 ura 3 mutants by pairing a URA3-flanking primer, URA3F or URA3R, with the $P B$-specific PBLR primer (Fig. 3d), followed by DNA sequencing analysis of the PCR products. We identified 4 different insertion sites in the URA3 locus (Fig. 3e). One was located in the promoter region, and all the others were located in the ORF. Also, $19 P B$ insertions occurred at TTAA, one at TTAG, and two at CTAA motifs.

We also performed an NGS comparison of the mutant pools before and after 5-FOA treatment. We obtained 3,266,688 and $3,865,123$ read pairs of $P B$ insertion junctions from 5-FOAtreated and untreated samples respectively. Ranking the fold changes of genes after 5-FOA treatment, URA5 and URA3 occupied the first and second positions (Fig. 3f), exceeding the third by $\sim 10$ times. URA5 and URA3 also occupied the top eight places in the rank of fold changes of insertion sites after 5-FOA treatment (Supplementary Figure 2a). Consistently, inverse PCR and sequencing analysis of 5-FOA resistant clones \#4, \#9, \#15, and \#26 confirmed $\mathrm{PB}$ [HIS1] insertion at the same position in URA5 ORF (Fig. 3g). Deletion of URA5 was found previously to cause partial resistance to 5 -FOA in $S$. cerevisiae ${ }^{25}$. However, deleting URA5 in both haploid and diploid C. albicans caused comparable 5-FOA resistance as deleting URA3 (Supplementary Figure 2b). Ura3 and Ura5 catalyze two consecutive steps in the de novo biosynthesis of pyrimidines ${ }^{26}$. Taken together, this proof-of-principle screen demonstrated the superb power of this $P B$-mediated mutagenesis system in identifying genes required for a cellular process.

Genome-wide genetic screening for fluconazole-resistant genes. To identify new antifungal resistance mechanisms, we applied the $P B$-transposon mutagenesis system to perform genome-wide profiling of genes whose inactivation causes resistance to fluconazole. The rationale of the screen is: if $P B$ insertion into a gene causes resistance to fluconazole, cells carrying this mutation will outcompete sensitive cells when grown in the presence of fluconazole and become overrepresented in the population. Thus, the subsequent NGS reads of $P B$ insertions in this gene in the pooled genomic DNA will increase correspondingly. The $P B$ insertion library was prepared by first growing YW02 cells in YPD+Dox medium and then dividing the culture into two equal parts for further cultivation in Dox-free medium with or without fluconazole. Cells were harvested after doubling 10 times and processed for NGS analysis.

We detected, in three independent experiments, an average of 5250 and 5122 genes from fluconazole-treated and untreated samples and ranked the genes according to the levels of enrichment after fluconazole treatment. Among the top 10 were three genes that either had been linked to azole susceptibility or are involved in ergosterol biosynthesis including ERG3, ERG6, and ERG251 (Fig. 4a and Supplementary Data 2). We randomly selected 10 genes ranked higher than $P M C 1$, which is the lowest ranking known resistant genes in our list, to construct haploid deletion mutants and found that they all showed increased resistance to fluconazole (Fig. 4b). Gene Ontology (GO) analyses of the genes that showed a significant increase in abundance in fluconazole-treated cells ( $\log 2$ fold change $>1$ and $p$-value $<0.05$ ) revealed an overrepresentation of genes associated with ergosterol and sphingolipid biosynthesis (Fig. 4c). We also performed Gene Set Enrichment Analysis (GSEA) and found significant enrichment of genes associated with ergosterol and sphingolipid biosynthesis in fluconazole-treated cells in the leading edge subset that has the highest correlation with fluconazole resistance (Fig. 4d). The latter group included FEN1, FEN12, and ARV1 that play important roles in sphingolipid biosynthesis and trafficking $^{27,28}$. Like ergosterol, sphingolipids are the main components of the fungal plasma membrane which is both the target of azole and polyene classes of antifungal drugs and site of drug transporters. We reasoned that the discovery of these genes in our screen likely reflects some previously unknown mechanisms of drug resistance. Thus, we further characterized FEN1 and FEN12, both encoding fatty-acid elongases that synthesize verylong-chain fatty acids as precursors of sphingolipid biosynth$e^{e s i s}{ }^{28}$. We verified that $f e n 1 \Delta / \Delta$ and fen $12 \Delta / \Delta$ mutants constructed in the diploid BWP17 background also exhibited significantly higher resistance to fluconazole than the WT strain (Fig. 4e).

fen $\Delta / \Delta$ mutants can tolerate higher levels of the toxic sterol. Since blocking DMCDD synthesis by inactivating ERG3 is a known resistance mechanism to fluconazole ${ }^{29}$, we quantified 
a

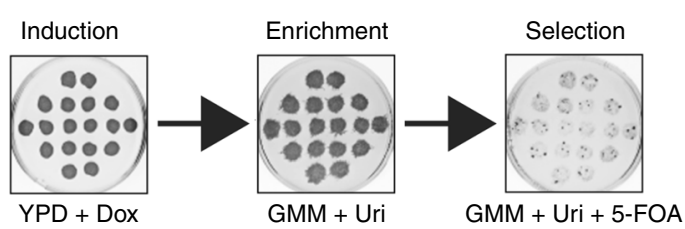

d

b $\mathrm{GMM}$

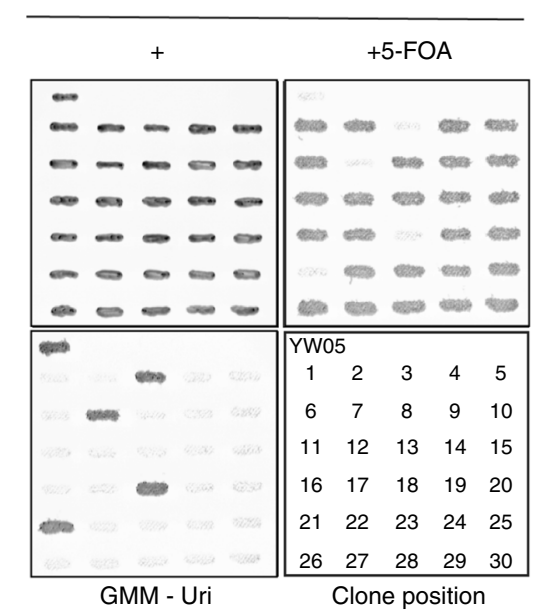

C
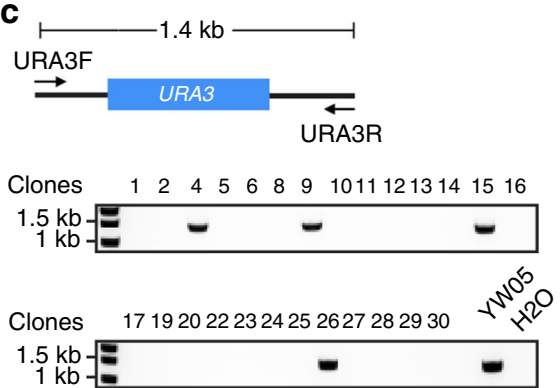

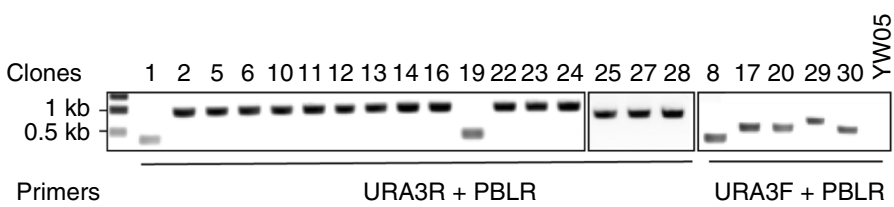

e
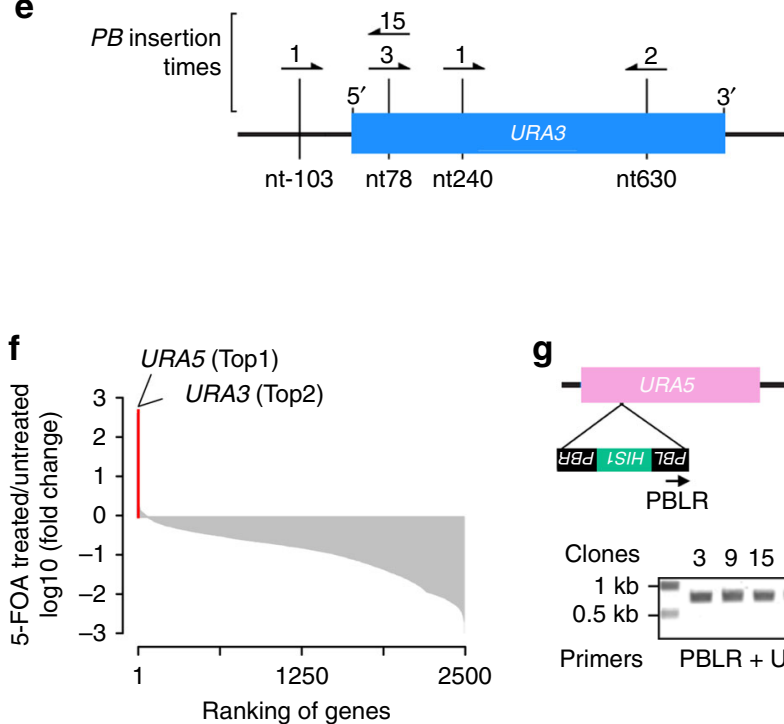

g

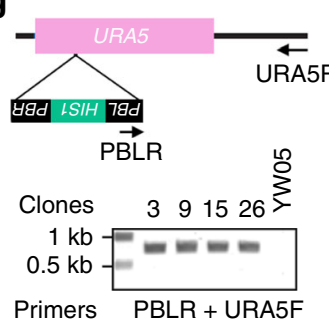

Fig. 3 Genetic screen for 5-FOA-resistant mutants caused by $P B$ transposition. a An illustration of the screening procedure. $\mathbf{b}$ Confirmation of the phenotype of 5-FOA-resistant mutants. Strains grown on a GMM+Uri+Arg+His (+) plate were replica-transferred onto a GMM+Uri+5-FOA (+5-FOA) or GMM+Arg+His (GMM-Uri) plate and then incubated at $30^{\circ} \mathrm{C}$ overnight. YW05 was included as a control. The lower right panel shows the strain layout. c Characterization of 5-FOA-resistant mutants by colony PCR. Arrows indicate the primers used to detect URA3. d PCR identification of PB insertion sites at the URA3 locus of the true 5-FOA-resistant clones from $\mathbf{b}$. Arrows indicate the primers used. e PB insertion sites identified at the URA3 locus. Sequence analysis identified four different sites indicated by vertical lines. Arrows denote $P B$ insertions, and the direction of arrows indicate the direction of HIS1 transcription in PB. The numbers indicate the number of mutants associated with each insertion site. $\mathbf{f}$ Fold changes of genes with $P B$ inserts after growing the mutant library in media containing 5-FOA in comparison with the same library grown in normal media. The top two genes are highlighted in red. $\mathbf{g}$ PCR identification of $P B$ insertion sites at the URA5 locus. Arrows denote the primers used

ergosterol and DMCDD in whole cell lysates and the plasma membrane using LC-MS. Due to the lack of standard DMCDD, we included an $\operatorname{erg} 3 \Delta$ strain to ensure correct identification of this molecule by combining the detected molecular mass and the absence of the compound in erg3 $\Delta$ cells (Fig. 5a, top and Supplementary Figure 3). LC-MS detected a $\sim 90 \%$ decrease of ergosterol in the whole cell lysates of both $f e n 1 \Delta / \Delta$ and $f e n 12 \Delta / \Delta$ mutants compared to WT controls grown in drug-free media (Fig. 5a, middle), indicating that lacking either FEN1 or FEN12 impairs ergosterol biosynthesis severely. As expected, fluconazole treatment caused a dramatic drop of ergosterol levels in all strains. Unexpectedly, we detected a $\sim 270 \%$ increase in the amount of DMCDD in both $f e n 1 \Delta / \Delta$ and $f e n 12 \Delta / \Delta$ cell lysates compared with WT cells upon fluconazole treatment (Fig. 5a, bottom). However, the DMCDD level in the plasma membrane fraction of $f e n 1 \Delta / \Delta$ and $f e n 12 \Delta / \Delta$ cells was found to be 35 and $29 \%$ of that in WT cells (Fig. 5a, bottom). The results suggest that, unlike $\operatorname{erg} 3 \Delta / \Delta$, the higher fluconazole resistance of $f e n \Delta / \Delta$ mutants is not due to decreased production of the toxic sterol.

Significant changes in sphingolipid composition in $f e n \Delta / \Delta$ mutants. As ergosterol and DMCDD insert themselves into the cell membrane mainly through interaction with sphingolipids ${ }^{30}$, we speculated that changes of the sphingolipid profile in fen $1 \Delta / \Delta$ and fen $12 \Delta / \Delta$ might be responsible for the tolerance of DMCDD. To test this hypothesis, we performed lipidomics profiling by LCMS. We detected rather different fatty-acid profiles between fen $1 \Delta / \Delta$ and $f e n 12 \Delta / \Delta$ mutants (Fig. $5 \mathrm{~b}$ and Supplementary Figure 4). The levels of fatty acids with 24 and 26 carbons decreased by $74-85 \%$ in both mutants grown in drug-free media (Fig. 5b), consistent with the loss of the very-long-chain fatty acid elongase. Varying degrees of increase or decrease were observed in the levels of fatty acids with 14-22 carbons; for instance, fen $1 \Delta /$ $\Delta$ cells grown in drug-free media showed $10-60 \%$ decrease in 


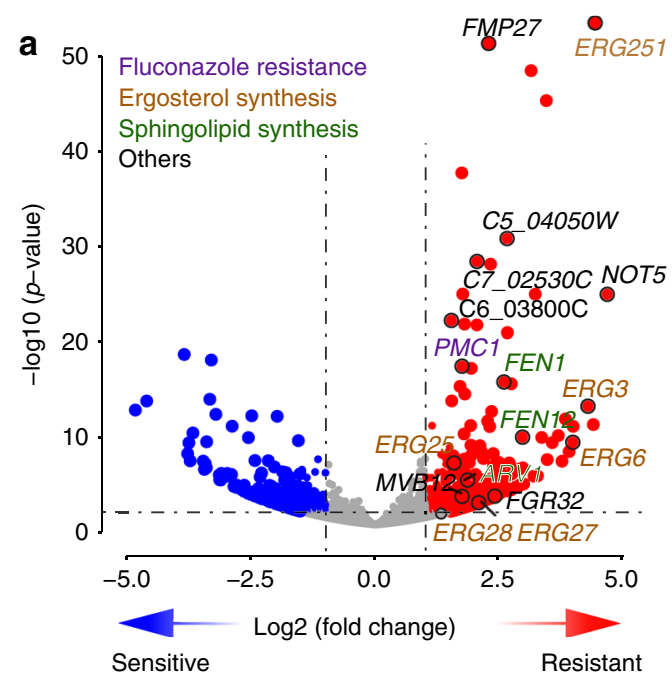

C

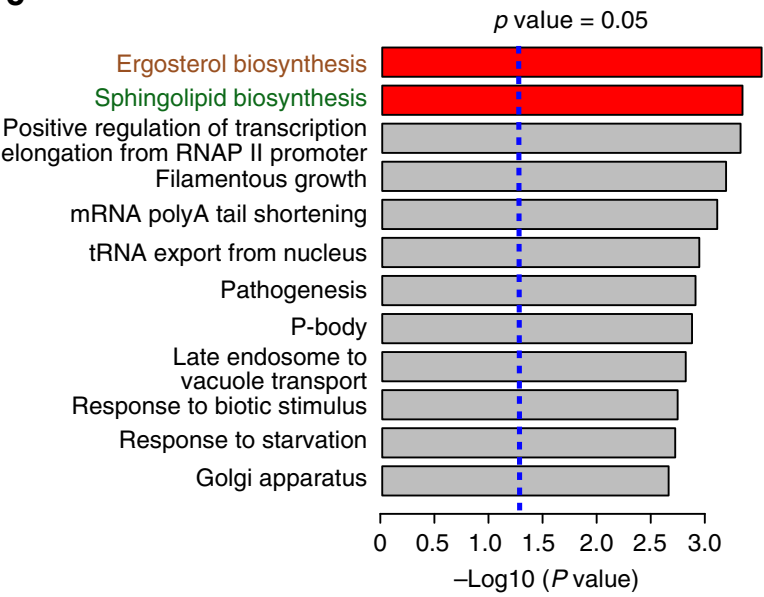

b

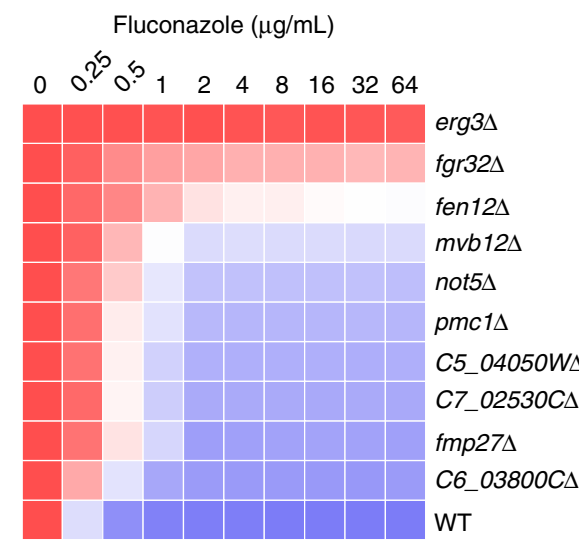

$\%$ Relative growth

0255075100

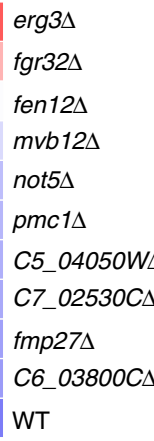

e

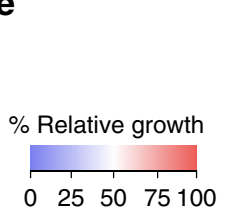

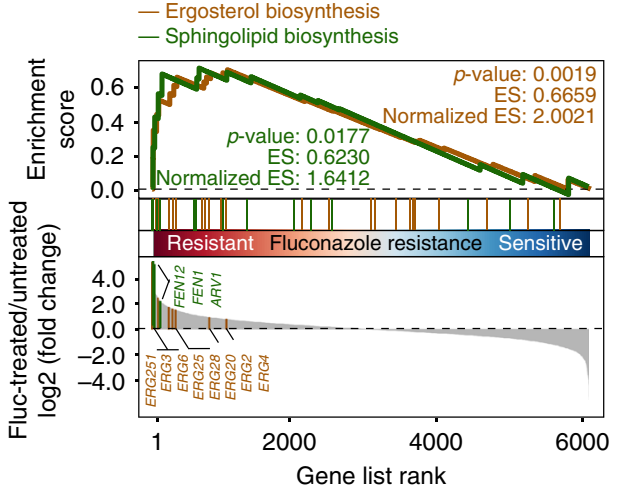

Fluconazole $(\mu \mathrm{g} / \mathrm{mL})$
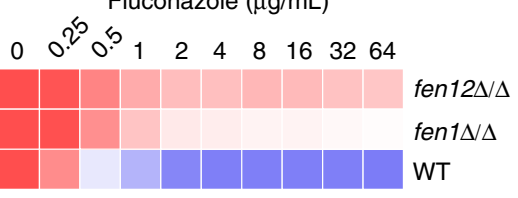

Fig. 4 Genetic screens for fluconazole-resistant mutants. a Volcano plot of fold changes of genes with $P B$ inserts in $P B$ transposition mutants grown in media containing $100 \mu \mathrm{g} / \mathrm{mL}$ fluconazole in comparison with the same mutants grown in normal media. $p$-Values were calculated using the Wald test. Ten candidates selected for phenotype confirmation are indicated. Genes known to be associated with fluconazole resistance and ergosterol and sphingolipid biosynthesis are shown with different colors. $\mathbf{b}$ Gene deletion of 10 candidates randomly selected from the top-ranked genes in a confirms increased resistance to fluconazole. Fluconazole susceptibility was evaluated using the standard CLSI broth microdilution protocol67. Cultures were incubated for 24 h, and growth was measured as $\mathrm{OD}_{600}$ and expressed as a percentage of the growth in the control wells with no drug (DMSO alone) for each strain. $\mathbf{c}$ Gene Ontology (GO) analysis of the PB-mutated genes that led to statistically significant fold increases $(p<0.05)$ after fluconazole treatment. Red bars highlight the ergosterol and sphingolipid biosynthesis pathway. $p$-Values were from Fisher's exact test. $\mathbf{d}$ Gene Set Enrichment Analysis (GSEA) of the NGS data from a. $p$-Values were determined using the Kolmogorov-Smirnov test. e Homozygous deletion of FEN1 or FEN12 in BWP17 also results in increased fluconazole resistance. The assay was done as described in $\mathbf{b}$

these fatty acids while $f e n 12 \Delta / \Delta$ cells showed $164 \%$ and $419 \%$ increase in FA(20:0) and FA(22:0), respectively (Fig. 5b). Similar fatty-acid profiles were detected in fluconazole-treated cells. Consistently, we detected a marked decrease in the levels of $\alpha$ hydroxyphytoceramide ( $\alpha \mathrm{HPC}$ ), inositolphosphoceramide (IPC), and mannosylinositolphosphoceramide (MIPC) species with a 26-carbon fatty acid chain in both $f e n 1 \Delta / \Delta$ and $f e n 12 \Delta / \Delta$ cells grown in drug-free media (Fig. 5b and Supplementary Figures 5, 6 and 7); in particular, there was a 20-fold decrease of d18:0/26:0 aHPC and 50-fold decrease of both d18:0/26:0 IPC and d18:0/ 26:0 MIPC in fen $12 \Delta / \Delta$ cells. In contrast, several $\alpha$ HPC, IPC, and MIPC molecules with a 20-24-carbon fatty acid chain showed an increase over a broad range; for example, d16:0/24:0 aHPC increased by 37.4 fold, 18:0/22:0 or d16:0/24:0 (the two molecules have identical mass) IPC by 73.3 fold, and d18:0/22:0 or d16:0/ 24:0 MIPC by almost 300 fold in fen $12 \Delta / \Delta$ cells (Fig. 5b). Strikingly, in response to fluconazole treatment, all species of aHPC and MIPC increased significantly in WT cells from 5.48 to 99.3 fold. This fluconazole-induced increase was more dramatic in fen $\Delta / \Delta$ cells; for example, d16:0/20:0 aHPC increased by 50.5 fold in $f e n 1 \Delta / \Delta$ and d16:0/24:0 aHPC increased by 361 fold in $f e n 12 \Delta /$ $\Delta$ cells. The most significant changes occurred in d18:0/22:0 or d16:0/24:0 and d18:0/20:0 or d16:0/22:0 MIPC which increased by $>3$ orders of magnitude (Fig. $5 b$ ). The results suggest that increasing the cellular levels of some species of sphingolipids might be a natural stress response to fluconazole. We speculate that high levels of sphingolipids could improve cell membrane strength leading to higher tolerance of the toxic sterol. 
a
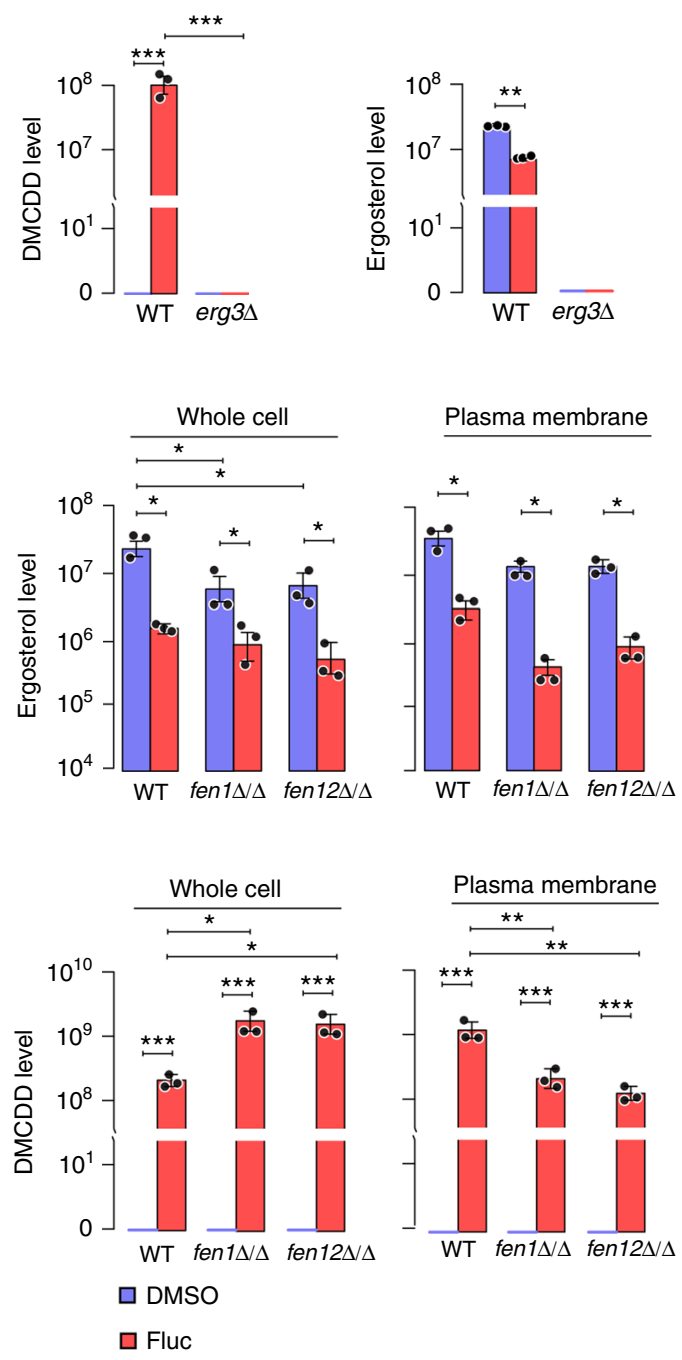

b

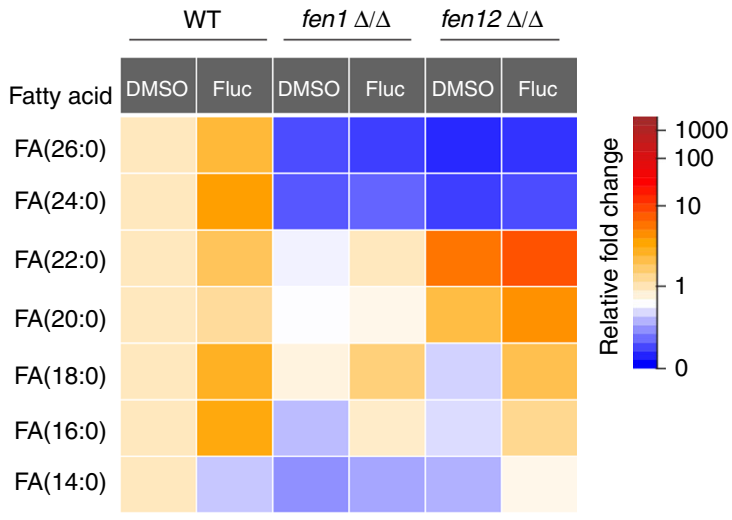

$\alpha \mathrm{HPC}$

Cer(d18:0/26:0 + 2O) + H

Cer(d16:0/26:0 + 2O) + H

Cer(d16:0/24:0 + 2O) + H

Cer(d16:0/22:0 + 2O) + H

Cer(d16:0/20:0 + 2O) + H

IPC

IPC (d18:0/26:0 + 2O) $-\mathrm{H}$

IPC(d18:0/24:0 + 2O) - H IPC (d16:0/26:0 + 2O) $-\mathrm{H}$ IPC(d18:0/22:0 + 2O) - H IPC(d16:0/24:0 + 2O) - H IPC(d18:0/20:0 + 2O) $-\mathrm{H}$ IPC(d16:0/22:0 + 2O) - H

MIPC

MIPC(d18:0/26:0 + 2O) - H

MIPC(d18:0/24:0 + 2O) - H MIPC(d16:0/26:0 + 2O) - H MIPC(d18:0/22:0 + 2O) - H MIPC(d16:0/24:0 + 2O) - H MIPC(d18:0/20:0 + 2O) - H MIPC(d16:0/22:0 + 2O) - H MIPC(d18:0/18:0 + 2O) - H MIPC(d16:0/20:0 + 2O) - H
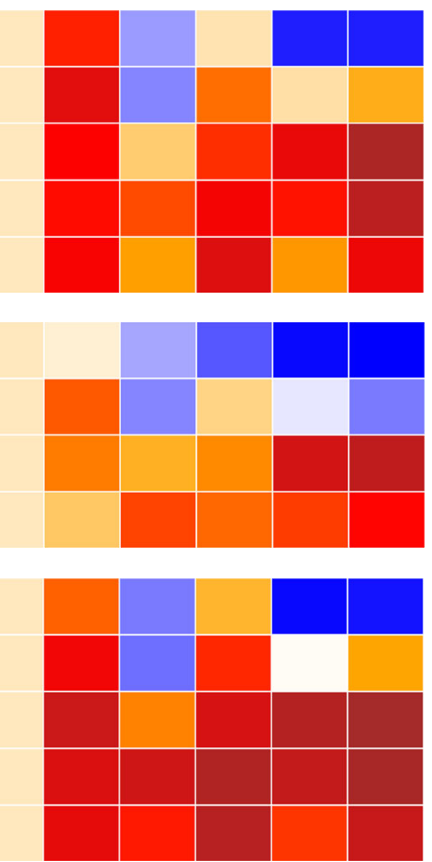

Fig. 5 LC-MS analysis of sterols and lipids in WT and fen $\Delta / \Delta$ cells. a LC-MS comparison of ergosterol and DMCDD levels in whole cell lysates and the plasma membrane fraction of WT (GZY803 and BWP17), erg3 $\Delta$, fen $1 \Delta / \Delta$, and fen $12 \Delta / \Delta$ cells with or without fluconazole treatment. Error bars represent s.d. from the mean of three independent experiments. Significance was measured using unpaired $t$-test $\left({ }^{\star} p<0.05,{ }^{\star \star} p<0.01,{ }^{\star \star \star} p<0.001\right)$. $\mathbf{b} \mathrm{LC}-\mathrm{MS}$ analysis of free fatty acids, $\alpha \mathrm{HPC}$, IPC, and MIPC sphingolipids in WT, fen $1 \Delta / \Delta$, and fen $12 \Delta / \Delta$ cells with or without fluconazole treatment

Mechanisms of sphingolipid overproduction in $f e n \Delta / \Delta$ mutants. We further investigated the mechanisms behind the fluconazole-induced sphingolipid overproduction. Fungal cells possess a remarkable capacity to counterbalance a disturbance to maintain cellular homeostasis. In response to fluconazole treatment, $C$. albicans reacts by overexpressing UPC2 which encodes a transcription factor that regulates ergosterol biosynthesis (Fig. 6a summarizes the biosynthetic pathways of ergosterol and sphingolipids) and sterol uptake ${ }^{31}$. Indeed, we detected the overexpression of UPC2, ERG3, and ERG11 in both WT and fen $\Delta / \Delta$ strains after fluconazole treatment (Fig. 6b). UPC2 also regulates genes involved in sphingolipid biosynthesis ${ }^{32-35}$. Consistently, we detected overexpression of the ceramide synthase gene LAG1, the IPC synthase gene AUR1, the MIPC synthase gene MIT1, and the $\mathrm{M}(\mathrm{IP})_{2} \mathrm{C}$ synthase gene IPT1 upon fluconazole treatment (Fig. 6b). Importantly, the fluconazole-induced UPC2 overexpression is much stronger in $f e n \Delta / \Delta$ cells than in WT cells. The results are consistent with the higher amounts of sphingolipids found in the mutants grown in the presence of fluconazole.
To determine whether the upregulation of sphingolipid biosynthesis is responsible for the increased resistance to fluconazole in $f e n 1 \Delta / \Delta$ and $f e n 12 \Delta / \Delta$ mutants, we attempted to delete $U P C 2$ and $L A G 1$ in $f e n 1 \Delta / \Delta$ and $f e n 12 \Delta / \Delta$ strains. Despite repeated efforts, we could not delete both copies of $L A G 1$ in either mutant and those of UPC2 in fen $1 \Delta / \Delta$, suggesting synthetic lethality. However, we found that deleting one copy of LAG1 or one copy of UPC2 in $f e n 1 \Delta / \Delta$ and $f e n 12 \Delta / \Delta$ appeared to increase their sensitivity to fluconazole (Fig. 6c). Also, deleting both copies of UPC2 in fen $12 \Delta / \Delta$ abolished its resistance to fluconazole (Fig. 6c). The results suggest that Upc2-induced upregulation of sphingolipid biosynthesis may underlie the increased fluconazole resistance of $f e n 1 \Delta / \Delta$ and fen $12 \Delta / \Delta$ mutants. Consistently, the fluconazole-induced upregulation of ERG3, ERG11, LAG1, AUR1, $M I T 1$, and IPT1 was significantly reduced or abolished when one or both copies of UPC2 was deleted (Fig. 6b). Together, the results corroborate the hypothesis that $f e n 1 \Delta / \Delta$ and fen $12 \Delta / \Delta$ mutants increase the cellular levels of sphingolipids in response to fluconazole by strongly upregulating the expression of genes 
a
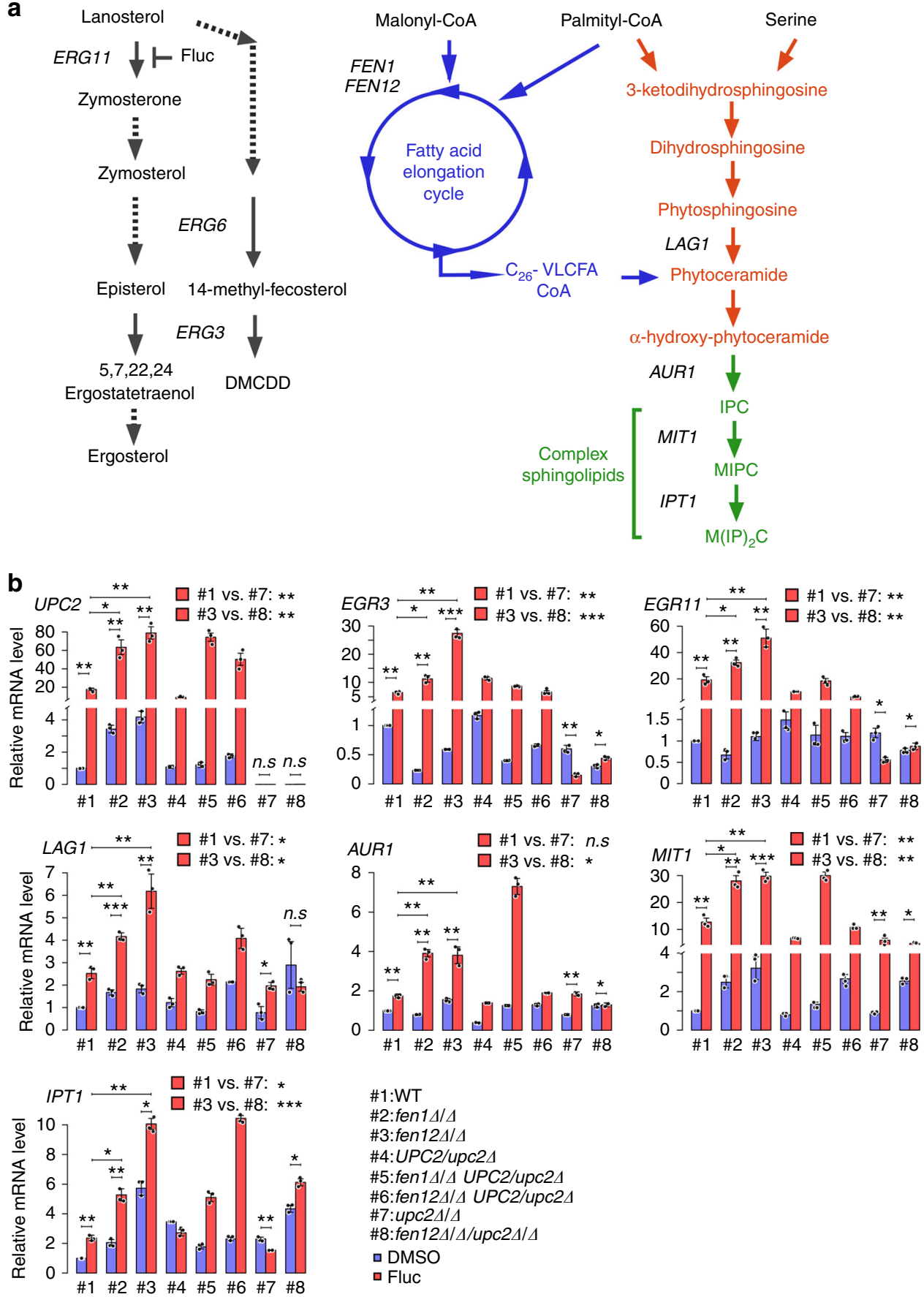

\#1:WT

\#2:fen 14/4

\#3:fen 124/4

\#4:UPC2/upc24

\#5:fen14/4 UPC2/upc24

\#6:fen124/4 UPC2/upc24

\#7:upc24/4

\#8:fen124/4/upc24/4

$\square$ DMSO

$\square$ Fluc

C
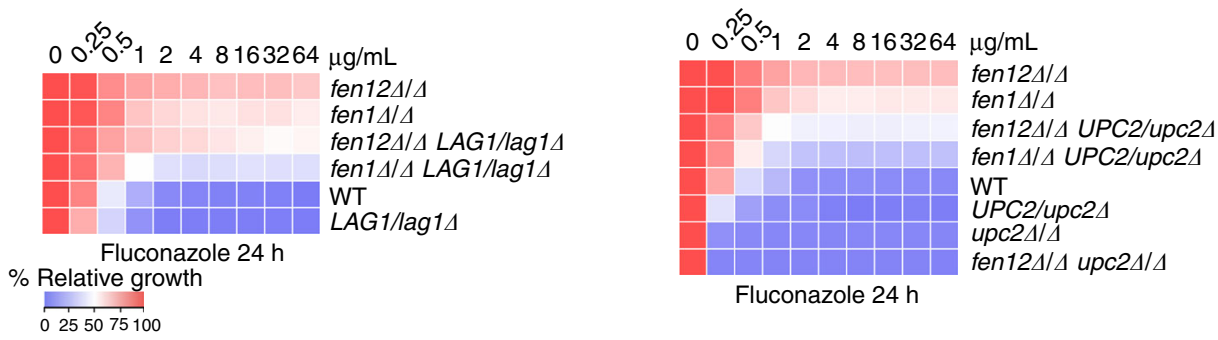

Fluconazole $24 \mathrm{~h}$

Fig. 6 Sphingolipid overproduction underlies fluconazole resistance of fen $\Delta / \Delta$ cells. a Illustration of ergosterol and sphingolipid biosynthesis pathways. b qPCR analysis of UPC2, ERG3, ERG11, LAG1, AUR1, MIT1, and IPT1 expression in WT, fen1 $\Delta / \Delta$, and fen $12 \Delta / \Delta$ mutants with UPC2 single or double knockout in the presence or absence of fluconazole. Error bars represent s.d. from the mean of three independent experiments. Significance was measured using unpaired $t$-test $\left({ }^{\star} p<0.05,{ }^{\star \star} p<0.01,{ }^{\star \star \star} p<0.001\right.$, n.s., not significant). c Fluconazole susceptibility of WT and the indicated mutants was evaluated using the standard CLSI broth microdilution protocol. Following $24 \mathrm{~h}$ of incubation, growth was measured as $\mathrm{OD}_{600}$ and expressed as a percentage of the growth in the control wells with no drug (DMSO alone) for each strain 
involved in sphingolipid biosynthesis, leading to tolerance of the toxic sterol.

\section{Discussion}

In recent years, considerable efforts have been made to introduce transposon mutagenesis strategies to create libraries of heterozygous $^{36,37}$ or homozygous ${ }^{38,39}$ mutants in C. albicans. In general, these methods start with in vitro transposition of a transposon-containing cassette, Tn5-UAU1 or Tn7-UAU1, into a C. albicans genomic DNA library 36,37 . Then, the mutagenized library is transformed into Escherichia coli for amplification before transformation into C. albicans. These methods are tedious, labor-intensive, and unsuitable for high-throughput genome-wide genetic studies. Also, their use in diploid C. albicans creates heterozygous mutations that do not result in readily detectable phenotypes except for a small number of haploinsufficient genes ${ }^{40,41}$. Mielich et al. recently introduced the Activator/Dissociation transposon of maize into haploid C. albicans $^{42}$, but the system is not very useful at its current state because of frequent autodiploidization.

Here, we introduce a simple and efficient $P B$ transposon-based mutagenesis system in haploid $C$. albicans, which has the following advantageous features. First, a single transposon insertion in the promoter or ORF of a gene is, in most cases, sufficient to inactivate a gene and produce a phenotype. Second, the transposon donor is inserted into a selectable marker gene, i.e., ARG4, and the transposase is expressed from a different locus under the control of the Tet-On promoter. Thus, a mutant library can be generated merely by growing the cells in a medium containing Dox to express the transposase and then selecting Arg + transposants in -Arg media. Third, transposition occurs at high frequencies and in a random manner. Our NGS analysis showed an even distribution of $P B$ insertions on all chromosomes and identified $P B$ inserts in $\sim 5000$ genes (Fig. 2e). Fourth, each mutant cell contains a single $P B$ insertion, which allows straightforward mapping of the affected gene by inverse PCR and DNA sequencing. Fifth, the precise excision of $P B$ from the insertion site restores the coding sequence allowing easy confirmation of the phenotype-genotype relationship. Our successful identification of URA3 and URA5 in 26 out of 30 random 5-FOAresistant mutants demonstrates the superb power of the $P B$ transposon mutagenesis system (Fig. 3). Furthermore, the $P B$ mediated mutagenesis system is readily applicable to wholegenome epistasis analysis of gene networks by first deleting a gene of interest before activating the transposition and screening for synthetic phenotypes. We anticipate that this $P B$ transposonbased mutagenesis platform will significantly accelerate discoveries to advance our understanding of the biology and pathogenesis of this pathogen.

Frequent isolation of multidrug-resistant Candida worldwide is a cause for concern because currently only three classes of drugs are available approved for the treatment of systemic fungal infections ${ }^{43,44}$. Hard lessons learned since the discovery of penicillin have taught us that microbial pathogens are extremely innovative in quickly finding tricks to defeat every one of our therapies. To win the war against infectious disease, it is imperative to elucidate how a pathogen has acquired resistance and even to predict how it can do it. The development of the $P B$ transposon mutagenesis system has allowed us to conduct genome-wide screening in C. albicans to identify genes underlying antifungal susceptibility. Our NGS analyses of $P B$ transposon insertion sites in mutants selected by growth in the presence of fluconazole in comparison with mutants grown in drug-free media revealed significant enrichment of genes involved in ergosterol biosynthesis, in particular, ERG3 and ERG6 whose mutation is well known to increase fungal resistance to azoles ${ }^{45-48}$. Deleting any one of 10 genes randomly picked from our resistant mutant list all led to increased fluconazole resistance (Fig. 4b), indicating that the screen was successful. Among the highest ranking genes were FEN1, FEN12, and ARV1 which play important roles in sphingolipid biosynthesis ${ }^{49,50}$. The understanding that sphingolipids are major components of fungal cell membrane and interact both physically and functionally with ergosterol $^{30,51,52}$, the target of azole and polyene antifungal drugs, prompted us to conduct a comprehensive analysis of FEN1 and FEN12. Our first informative finding was a severalfold increase in the cellular levels of the toxic sterol in both $f e n \Delta / \Delta$ mutants compared to WT cells (Fig. 5a), which indicated a resistance mechanism distinct from that of the erg3 mutant ${ }^{2,46}$. Interestingly, the level of the toxic sterol in the plasma membrane is significantly lower in the mutants than in the wild type (Fig. 5a), suggesting that the mutants pack fewer toxic sterol molecules into the membrane. Furthermore, we detected dramatic changes in the sphingolipid composition in whole cell extracts (Fig. 5b). The most striking change is the increase of more than 3 orders of magnitude of some MIPC species in fen $12 \Delta / \Delta$ cells upon fluconazole treatment, which also increased the total cellular level of sphingolipids. An illuminating observation was the sharp increase of the same MIPC species in WT cells after fluconazole treatment (Fig. 5b), indicating that C. albicans alters its sphingolipid composition as a natural stress response mechanism to the disruption of ergosterol biosynthesis. This could be a conserved survival strategy because eukaryotic cells are known to have a huge capacity to preferentially modify the cellular levels and make-up of sphingolipids in response to changes in sterol composition in their membrane as an adaptation mechanism to maintain membrane structure and function ${ }^{52}$. In S. cerevisiae, mutation of $E L O 3$, a homolog of FEN12, also affected sphingolipid composition significantly ${ }^{53}$. Many previous studies have revealed intricate relationships between ergosterol and sphingolipid biosynthesis and resistance to azoles. Particularly consistent with our findings, Prasad et al. found that blocking $\mathrm{M}(\mathrm{IP})_{2} \mathrm{C}$ synthesis by deleting the IPT1 gene significantly increased C. albicans susceptibility to azoles $^{54}$; furthermore, the level of the drug efflux pump Cdr1 in the plasma membrane was reduced in $i p t 1 \Delta / \Delta$ cells as well as in cells treated with fumonisin B1, an inhibitor which blocks the synthesis of phytoceramide ${ }^{55}$. Consistently, we demonstrated that blocking sphingolipid biosynthesis reduced the fluconazole resistance in fen $1 \Delta / \Delta$ and fen $12 \Delta / \Delta$ cells. Sphingolipids are abundant components of the fungal cell membrane and play both structural and signaling $\operatorname{roles}^{56}$. Numerous experiments have shown that changes of sphingolipid composition can dramatically alter the properties of cell membranes, such as rigidity, fluidity, and domain organization which further influences cellular functions $s^{57}$. Thus, the marked changes of the cellular sphingolipid composition in $f e n \Delta / \Delta$ mutants might lead to fluconazole resistance by reducing the incorporation of the toxic sterol into the cell membrane and limiting subsequent structural and functional damage of the cell membrane. Although we do not have direct evidence confirming repellence of the toxic sterol from the cell membrane by high levels of sphingolipids, this can be expected based on the chemical properties of sterols and sphingolipids and is indirectly supported by various previous studies. Cell membranes have a complex organization featuring domains with distinct lipid compositions known as rafts whose formation is determined by the hydrophobic interaction between sterols and sphingolipids ${ }^{58,59}$. However, the toxic sterol is structurally different from ergosterol in terms of the absence of B-ring unsaturation, the presence of the 6-OH group and the additional C-14 methyl group, and side-chain modification. In particular, the polar 6-OH group is thought to interfere with sterol-sphingolipid 
packing in the plasma membrane, causing membrane damage ${ }^{60,61}$. The high level of sphingolipids in fen $\Delta / \Delta$ mutants could strengthen the cell membrane by restraining membrane deformation. When the additional free energy required for incorporating polar groups of the toxic sterol cannot be compensated by membrane deformation, they must be pushed out to reach a lower energy status. Thus, more toxic sterol molecules with a polar side chain would be repelled from the membrane by the nonpolar chain of sphingolipids. Taken together, it is reasonable to believe that high levels of sphingolipids can repel toxic sterol molecules from the cell membrane. It was reported that fen $1 \Delta / \Delta$ and $f e n 12 \Delta / \Delta$ mutants exhibited increased sensitivity to amphotericin $\mathrm{B}^{62}$. It is widely accepted that amphotericin $\mathrm{B}$ binds to ergosterol in a parallel manner to form barrel-stave type pores and penetrate cell membranes, in which their hydrophilic polyhydroxy side is pointing inward to constitute the pore lining and their hydrophobic lipophilic heptaene part is directing outward to interact with the membrane interior ${ }^{63,64}$. While the high levels of sphingolipids in the membrane hinder the insertion of the hydrophilic toxic sterol, the binding of amphotericin B to ergosterol is not affected; and on the contrary, the shorter sphingolipids in $f e n \Delta / \Delta$ mutants could expose ergosterol molecules and thus facilitate the binding to amphotericin B, making mutant cells more sensitive to this drug. In future studies, it would be interesting to know whether high cellular levels of sphingolipids contribute to azole resistance in clinical isolates of C. albicans and other fungal pathogens.

\begin{abstract}
Methods
Strains, media, and growth conditions. All haploid C. albicans strains were

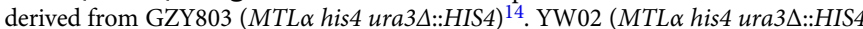
PTet-On-CaPBase::SAT1 arg4::PB[URA3]), and YW05 (MTL $\alpha$ his4 ura3A::HIS4 his1 $\triangle:: U R A 3$ PTet-On-CaPBase::SAT1 arg4::PB[HIS1]) were used as the parental strain for transposon mutagenesis. A complete list of strains and their genotypes are provided in Supplementary Table 2. C. albicans was routinely grown at $30^{\circ} \mathrm{C}$ in YPD medium (2\% yeast extract, $1 \%$ bactopeptone, and $2 \%$ glucose) or in GMM ( $2 \%$ glucose and $6.79 \mathrm{~g} / \mathrm{L}$ yeast nitrogen base without amino acids). 5-FOA (Sigma, F5013-250MG) was used at a concentration of $1 \mathrm{~g} / \mathrm{L}$ in GMM plates supplemented with uridine. Dox (Sigma, D9891-1G) was used in YPD medium at a final concentration of $50 \mu \mathrm{g} / \mathrm{mL}$ to induce the expression of CaPBase. Stock solutions of fluconazole (Sigma, F8929-100MG) was prepared in dimethyl sulfoxide (Sigma, V900090-500ML) and stored at $-20^{\circ} \mathrm{C}$ until use.
\end{abstract}

Plasmid construction. pPB[URA3] was constructed as follows: the URA3 marker was PCR-amplified from a pBluescript-based plasmid with primers URA3ApaI and URA3SacII (all primers used in this study are shown in Supplementary Data 1), and cloned into the ApaI and SacII sites of pPB[ura4] to generate pPB[URA3].

Construction of pARG4-PB[URA3]: the EcoRI-PstI fragment including the intact PB[URA3] transposon cassette was PCR-amplified from $\mathrm{pPB}$ [URA3] with primers PB[URA3]EcoRI and PB[URA3]PstI, and inserted into the EcoRI and PstI sites of a pBluescript-based plasmid containing ARG4 selectable marker.

Construction of pARG4-PB[HIS1]: the HIS1 marker was PCR-amplified from a pBluescript-based plasmid with primers HIS1ApaI and HIS1SalI, and cloned into the ApaI and SalI sites of pARG4-PB[URA3] to generate pARG4-PB[HIS1].

Construction of pNIM1-CaPBase: site-directed mutagenesis of the PBase coding sequence in plasmid pDUAL-PBase was performed using Quick-Change SiteDirected Mutagenesis Kit (Stratagene, 200514) to generate pDUAL-CaPBase which contains a codon-optimized PBase with hyperactivity. Then a PCR fragment containing the CaPBase was amplified from pDUAL-CaPBase with primers PBaseXhoI and PBaseBamHI, and cloned into the SalI and BglII sites of pNIM1 to generate pNIM1-CaPBase. pPB[ura4], pDUAL-PBase, and pNIM1 were described previously 20,65 .

Construction of strains YW02 and YW05. YW02 was constructed from GZY803 by introducing the linear DNA fragment from pNIM1-CaPBase containing the PTet-On-CaPBase fusion and the XhoI-NotI fragment from pARG4-PB[URA3] containing the ARG4-PB[URA3] fusion to transform GZY803 to become nourseothricin resistance and uridine prototrophic. Correct integration was confirmed by PCR.

YW05 was constructed from YW11 by introducing the linear DNA fragment from pNIM1-CaPBase containing the PTet-On-CaPBase fusion and the XhoI-NotI fragment from pARG4-PB[HIS1] containing the ARG4-PB[HIS1] fusion to transform YW11 to become nourseothricin resistance and histidine prototrophic Correct integration was confirmed by PCR.

Inverse PCR for mapping PB insertion sites. Genomic DNA was prepared using MasterPure Yeast DNA Purification Kit (Epicentre Technologies, MPY80200) and digested with BfuCI overnight. After heat inactivation of BfuCI, the digested genomic DNA was self-ligated with T4 DNA ligase at $16^{\circ} \mathrm{C}$ overnight. Primers used to recover the flanking sequence of the left side of the $P B$ transposon were PBLf and PBLr. PCR products were purified from agarose gel and sequenced using Seq1 as the sequencing primer. The sequencing results were used to BLAST-search Candida Genome Database (www.candidagenome.org) to determine the $P B$ insertion sites.

Quantitative PCR. To determine the copy number of $P B$ [URA3], genomic DNA was purified using MasterPure yeast DNA purification kit and analyzed on STRATAGENE Mx3000p using Maxima SYBR Green qPCR Master Mix (2X) with separate ROX vial (Thermo Fisher Scientific, K0253) according to the manufacturer's instructions. PCR was carried out with primers CDC28 sense and CDC28 antisense to amplify $C D C 28$, URA3 sense and URA3 antisense to amplify $P B$ [URA3]. $P B$ copy number was determined by $\triangle \triangle C T$ method using the genomic DNA of strain YW02 as a control, which has a single copy of $P B^{66}$. Since the ratios of $P B$ versus $C D C 28$ were usually not integral numbers, we estimated the copy number to be the integral closest to the ratios.

To detect the expression of CaPBase, ERG11, ERG3, UPC2, LAG1, AUR1, MIT1, and IPT1, total RNA was isolated using the RNeasy Mini Kit (QIAGEN, 74106) and RNase-Free DNase Set (QIAGEN, 79254) and then reverse-transcribed using the SuperScript ${ }^{\mathrm{mT}}$ II Reverse Transcriptase (Invitrogen, 18064014). Changes in transcript levels of target genes were analyzed using the Maxima SYBR Green qPCR Master Mix (2X) with separate ROX vial with primers PBase sense and PBase antisense for CaPBase, ERG11 sense and ERG11 antisense for ERG11, ERG3 sense and ERG3 antisense for ERG3, UPC2 sense and UPC2 antisense for UPC2, LAG1 sense and LAG1 antisense for LAG1, AUR1 sense and AUR1 antisense for AUR1, MIT1 sense and MIT1 antisense for MIT1, IPT1 sense and IPT1 antisense for IPT1, and normalized to CDC28 transcript levels using the $\Delta \Delta \mathrm{CT}$ method.

Transposition induction. In the experiments using integrated $P B[U R A 3]$ or $P B$ [HIS1] as the transposon donor, a single colony of YW02 or YW05 was first cultured in liquid YPD medium at $30^{\circ} \mathrm{C}$ overnight. Then, the cells were transferred to liquid or solid YPD medium supplemented with $50 \mu \mathrm{g} / \mathrm{mL}$ Dox to induce the expression of CaPBase and undergo transposition. To calculate transposition efficiency, aliquots of cells were harvested at timed intervals and spread on GMM plates to count the number of cells that had undergone transposition and on YPD plates to obtain the total number of cells. Transposition efficiency was calculated as the number of colonies on GMM plates divided by the number of colonies on YPD plates.

Flow cytometry. Flow cytometry analysis was performed using a BD FACSCalibur as described previously ${ }^{14,22}$. Mid-log phase cells were collected, washed, and fixed with $70 \%$ (vol/vol) ethanol (Sigma, E7023-500ML). Cells were washed with solution I (200 mM Tris-HCl, pH 7.5 and $20 \mathrm{mM} \mathrm{EDTA}$ ) once and treated with $10 \mathrm{mg} /$ mL RNase A (Sigma, R4875-100MG) (1:100 dilution in solution I). Cells were washed with PBS and resuspended in $5 \mathrm{mg} / \mathrm{mL}$ propidium iodide (Sigma, 8184525MG) (1:50 dilution in PBS) incubated in the dark at $4{ }^{\circ} \mathrm{C}$ overnight. Stained cells were collected and resuspended in PBS and sonicated before loading.

Mapping PB insertion sites by next-generation sequencing. A single colony of YW02 or YW05 cells were inoculated into $5 \mathrm{~mL}$ of YPD and grown at $30^{\circ} \mathrm{C}$ to the exponential phase. Eighteen equal aliquots of the culture, each containing about 10,000 cells, were spotted on a YPD plate containing $50 \mu \mathrm{g} / \mathrm{mL}$ Dox to induce CaPBase expression. After $24 \mathrm{~h}$ of incubation at $30^{\circ} \mathrm{C}$, cells on the induction plates were replica-transferred to a GMM plate without Dox to enrich the cells that had undergone transposition. The GMM plate was incubated at $30^{\circ} \mathrm{C}$ for 2 days before collecting the cells for the extraction of genomic DNA using the MasterPure Yeast DNA Purification Kit. Tagmentation was carried out by using the TruePrep DNA Library Prep Kit V2 for Illumina (Vazyme Biotech, TD501) according to the manufacturer's instructions. The first round of PCR was carried out to amplify $P B$ together with its flanking sequences using a $P B$-specific primer close to the insertion site, PBseq and a primer annealing to the Tn5 adaptor, N7XX. The cycling parameters were: $3 \mathrm{~min}$ at $72{ }^{\circ} \mathrm{C} ; 30 \mathrm{~s}$ at $98^{\circ} \mathrm{C} ; 12$ cycles of $15 \mathrm{~s}$ at $98{ }^{\circ} \mathrm{C}, 30 \mathrm{~s}$ at $60^{\circ} \mathrm{C}$, and $3 \mathrm{~min}$ at $72{ }^{\circ} \mathrm{C} ; 10 \mathrm{~min}$ at $72{ }^{\circ} \mathrm{C} ; 4^{\circ} \mathrm{C}$ forever. The second round of PCR was performed using NEBNext ${ }^{\oplus}$ Ultra ${ }^{\text {Dtw }}$ II ${ }^{\circ} 5^{\circledR}$ Master Mix (NEB, M0544L) to add sequences required for Illumina sequencing with an Illumina flowcell attachment primer, TruPBseq and partial Tn5 adaptor primer, Illumina-R. Cycling conditions are: $1 \mathrm{~min}$ at $98^{\circ} \mathrm{C} ; 7$ cycles of $10 \mathrm{~s}$ at $98^{\circ} \mathrm{C}, 75 \mathrm{~s}$ at $67^{\circ} \mathrm{C} ; 5 \mathrm{~min}$ at $67^{\circ} \mathrm{C}$; $4^{\circ} \mathrm{C}$ forever. The size selection and purification of the PCR products were carried out with AMPure XP beads (Beckman Coulter, A63882). 
Genetic screens using PB-mediated mutagenesis system. In the screen for 5FOA-resistant mutants, insertional mutagenesis in YW05 cells was induced by culturing on YPD plate containing Dox for $24 \mathrm{~h}$ and then replica-transferred to GMM plates containing uridine to select for Arg + His + cells. After incubation at $30^{\circ} \mathrm{C}$ for $24 \mathrm{~h}$, cells on the above GMM plates were replica-transferred to GMM plates supplemented with uridine and 5-FOA. The 5-FOA plates were then incubated at $30^{\circ} \mathrm{C}$ until resistant colonies had formed. For screening in the liquid medium, Arg+His + mutant cells were collected and washed three times with PBS. Two equal aliquots of cells were cultivated further in $20 \mathrm{~mL}$ GMM medium containing uridine with or without 5 -FOA at $30^{\circ} \mathrm{C}$ for $48 \mathrm{~h}$; then cells were harvested and processed for NGS analysis.

In the screen for fluconazole-resistant mutants, transposition-mediated mutagenesis was performed using strain YW02 grown in YPD plate containing Dox for $24 \mathrm{~h}$ at $30^{\circ} \mathrm{C}$. Then, the cells were replica-transferred to GMM plates and incubated at $30^{\circ} \mathrm{C}$ for $24 \mathrm{~h}$ to enrich $\mathrm{Arg}+\mathrm{Ura}+$ cells that had undergone the transposition. Then, mutant cells were collected and washed three times with PBS. Approximately $2-4 \times 10^{6}$ cells $/ \mathrm{mL}$ were cultivated further in $20 \mathrm{~mL}$ YPD medium in the presence or absence of fluconazole. Cell growth was monitored by measuring $\mathrm{OD}_{600}$, and cells were harvested after the population had doubled 10 times and processed for NGS analysis.

Fluconazole susceptibility test. Antifungal susceptibility testing of the strains included in this study was performed by using the broth microdilution method described in the CLSI document M27-A $3^{67}$ in a 96-well plate format.

Fluconazole concentrations tested ranged from 64 to $0.25 \mu \mathrm{g} / \mathrm{mL}$. RPMI 1640 medium (Thermo Fisher Scientific, 21875109) was prepared according to the CLSI document. The medium was buffered with $0.165 \mathrm{M}$ morpholinepropanesulfonic acid (MOPS), and its $\mathrm{pH}$ was adjusted using $\mathrm{NaOH}$ and $\mathrm{HCl}$. Cell inoculum was $\sim 1 \times 10^{3}$ cells per well. Plates were incubated without shaking at $30^{\circ} \mathrm{C}$ for $24 \mathrm{~h}$. The content of each well was carefully resuspended by pipetting up and down before $\mathrm{OD}_{600}$ was measured using the Spark multimode microplate reader (TECAN, Switzerland).

Preparation of C. albicans plasma membrane. Highly purified plasma membrane was isolated as previously described for $S$. cerevisiae with minor modifications. $C$. albicans yeast cells were harvested by centrifugation $(5000 \mathrm{~g}, 5 \mathrm{~min})$, then washed twice with $0.4 \mathrm{M}$ sucrose (Sigma, V900116-500G) in buffer A ( $25 \mathrm{mM}$ imidazole, $\mathrm{pH} 7.0$ adjusted by $\mathrm{HCl}$ ). Enough $0.4 \mathrm{M}$ sucrose in buffer $\mathrm{A}$ and glass beads were added to the cell pellet and the mix was vortexed for 1 min 8-10 times, with 2 min on ice between rounds. Then the mix was centrifuged at $530 \mathrm{~g}$ (2500 rpm in Sorvall SS34 rotor) for $20 \mathrm{~min}$ to pellet unbroken cells and glass beads, and the supernatant was centrifuged at 22,000g (16,000 rpm in Sorvall SS34 rotor) for $30 \mathrm{~min}$ to obtain a pellet that includes the plasma membranes and mitochondria. The pellet was resuspended in buffer A by gentle vortexing for $30 \mathrm{~s}$ and the resuspended membranes were loaded onto discontinuous sucrose gradients (overlaying three $4 \mathrm{~mL}$ layers of $2.25,1.65$, and $1.1 \mathrm{M}$ sucrose in buffer A in a $14 \times 89 \mathrm{~mm}$ Beckman ultraclear tube) and centrifuged either overnight $(14 \mathrm{~h})$ at $80,000 \mathrm{~g}(22,000 \mathrm{rpm})$ or for $6 \mathrm{~h}$ at $284,000 \mathrm{~g}(40,000 \mathrm{rpm})$ in the Beckmann SW41 or SW40Ti rotor. The membrane band at the $2.25 / 1.65 \mathrm{M}$ sucrose interface is essentially pure plasma membrane, which was collected at these interfaces from the top of the gradient with a Pasteur pipette. The collected membrane was diluted four times with buffer A and pelleted at 30,000 $\mathrm{g}(18,000 \mathrm{rpm}$ Beckman $50 \mathrm{Ti}$ rotor) for $40 \mathrm{~min}$. The plasma membrane pellet was suspended in $80 \%$ methanol and stored at $-80^{\circ} \mathrm{C}$ for sterol analysis.

Sterol analysis. Sterols were extracted using pre-chilled $80 \%(\mathrm{v} / \mathrm{v})$ methanol (Sigma, 34860-1L-R) according to the method of Yuan et al. ${ }^{68}$. C. albicans strains were cultured in YPD medium with or without fluconazole for $24 \mathrm{~h}$, and the PBS washed cells were harvested by centrifugation. Add pre-chilled $80 \%(\mathrm{v} / \mathrm{v})$ methanol and glass beads to the cell pellets. Vortex $1 \mathrm{~min}$, then keep on ice for $2 \mathrm{~min}$. Repeat this step 8 or 10 more times. Centrifuge at $14,000 \mathrm{~g}$ for $40 \mathrm{~min}$ using a refrigerated centrifuge at $4{ }^{\circ} \mathrm{C}$ to obtain the supernatant. The extracts were vacuum-dried and stored at $-80^{\circ} \mathrm{C}$. The dried samples were solubilized in methanol prior to analysis by LC-MS. Equal amounts of cells were processed for protein extraction, and protein concentration was measured by Pierce ${ }^{\mathrm{m}}$ BCA Protein Assay Kit (Thermo Fisher Scientific, 23227) to conduct normalization.

LC-MS analysis was performed with a UPLC system, which was coupled to a QExactive orbitrap mass spectrometer (Thermo Fisher, CA) equipped with an atmospheric pressure chemical ionization (APCI) probe. Sterol extracts were separated by a Kinetex ${ }^{\oplus} 100 \times 2.1 \mathrm{~mm} 2.6 \mu \mathrm{m}$ column (Phenomenex). A binary solvent system was used, in which mobile phase A consisted of $100 \% \mathrm{H}_{2} \mathrm{O}(0.1 \%$ FA), $10 \mathrm{mM}$ ammonium acetate, and mobile phase B of $100 \% \mathrm{ACN}(0.1 \% \mathrm{FA})$. A 10 -min gradient with a flow rate of $300 \mu \mathrm{L} / \mathrm{min}$ was used. Column chamber and sample tray were held at 45 and $10{ }^{\circ} \mathrm{C}$, respectively. Data with mass ranges of $\mathrm{m} / \mathrm{z}$ 70-150 was acquired at a positive ion mode with data dependent MSMS acquisition. The full scan and fragment spectra were collected with a resolution of 70,000 and 17,500, respectively. The source parameters are as follows: discharge current: $6 \mu \mathrm{A}$; capillary temperature: $350^{\circ} \mathrm{C}$; heater temperature: $300^{\circ} \mathrm{C}$; sheath gas flow rate: 35 Arb; auxiliary gas flow rate: 15 Arb. Data analysis was performed by the software Xcalibur (Thermo Fisher, CA). Compounds were identified based on their retention times and the masses detected in the liquid chromatography-mass spectrometry system.

Lipid analysis. Lipids were extracted according to the method of Bligh and Dyer ${ }^{69}$. C. albicans strains were cultured in YPD medium with or without fluconazole for $24 \mathrm{~h}$, and the PBS washed cells were harvested by centrifugation. The cell pellets were lysed in PBS by bead-beating mechanical disruption at $4{ }^{\circ} \mathrm{C}$. The supernatants were then extracted with chloroform (AMRESCO, 0757-500ML)/methanol (2:1) at a final ratio of $20 \%(\mathrm{v} / \mathrm{v})$. Vortex $30 \mathrm{~s}$, then keep for $2 \mathrm{~min}$. Repeat this step 5 more times. Centrifuge at $3000 \mathrm{~g}$ for $20 \mathrm{~min}$ using a refrigerated centrifuge at $4{ }^{\circ} \mathrm{C}$ to obtain the supernatant. The extracts were evaporated to dryness under $\mathrm{N}_{2}$ at room temperature and stored at $-80{ }^{\circ} \mathrm{C}$. The dried samples were solubilized in dichloromethane (Sigma, 650463-1L) methanol (2:1) before analysis by LC-MS Equal amounts of cells were processed for protein extraction and protein concentration was measured by BCA assay to conduct normalization.

LC-MS analysis was performed with a UPLC system was coupled to a QExactive orbitrap mass spectrometer (Thermo Fisher, CA) equipped with a heated electrospray ionization (HESI) probe. Lipid extracts were separated by a CORTECS C18 $100 \times 2.1 \mathrm{~mm} 1.9 \mu \mathrm{m}$ column (Waters). A binary solvent system was used, in which mobile phase A consisted of $\mathrm{ACN}: \mathrm{H}_{2} \mathrm{O}(60: 40), 10 \mathrm{mM}$ ammonium acetate, and mobile phase B of IPA:ACN (90:10), $10 \mathrm{mM}$ ammonium acetate. A 35-min gradient with a flow rate of $220 \mu \mathrm{L} / \mathrm{min}$ was used. Column chamber and sample tray were held at 45 and $10{ }^{\circ} \mathrm{C}$, respectively. Data with mass ranges of $\mathrm{m} / \mathrm{z}$ $240-2000$ and $m / z 200-2000$ was acquired at positive ion mode and negative ion mode with data dependent MSMS acquisition. The full scan and fragment spectra were collected with a resolution of 70,000 and 17,500, respectively. The source parameters are as follows: spray voltage: $3000 \mathrm{~V}$; capillary temperature: $320^{\circ} \mathrm{C}$; heater temperature: $300^{\circ} \mathrm{C}$; sheath gas flow rate: $35 \mathrm{Arb}$; auxiliary gas flow rate: 10 Arb. Data analysis and lipid identification were performed by the software Lipidsearch 4.0 (Thermo Fisher, CA). Compounds were identified based on their retention times and the masses detected in the liquid chromatography-mass spectrometry system.

\section{Data availability}

The sequencing data were deposited to NCBI under BioProject ID PRJNA486232. The authors declare that all other data supporting the findings of this study are available within the article and its Supplementary Information files, or from the corresponding authors upon request.

Received: 1 May 2018 Accepted: 3 October 2018

Published online: 29 October 2018

\section{References}

1. Pfaller, M. A. \& Diekema, D. J. Epidemiology of invasive candidiasis: a persistent public health problem. Clin. Microbiol. Rev. 20, 133-163 (2007).

2. Sanglard, D. Emerging threats in antifungal-resistant fungal pathogens. Front. Med. (Lausanne) 3, 11 (2016)

3. Vanden Bossche, H., Koymans, L. \& Moereels, H. P450 inhibitors of use in medical treatment: focus on mechanisms of action. Pharmacol. Ther. 67, 79-100 (1995).

4. Cowen, L. E. \& Steinbach, W. J. Stress, drugs, and evolution: the role of cellular signaling in fungal drug resistance. Eukaryot. Cell 7, 747-764 (2008).

5. Sanglard, D., Ischer, F., Calabrese, D., Micheli, M. \& Bille, J. Multiple resistance mechanisms to azole antifungals in yeast clinical isolates. Drug Resist. Updat. 1, 255-265 (1998).

6. Roemer, T. et al. Large-scale essential gene identification in Candida albicans and applications to antifungal drug discovery. Mol. Microbiol. 50, 167-181 (2003).

7. Homann, O. R., Dea, J., Noble, S. M. \& Johnson, A. D. A phenotypic profile of the Candida albicans regulatory network. PLoS Genet. 5, e1000783 (2009).

8. Noble, S. M., French, S., Kohn, L. A., Chen, V. \& Johnson, A. D. Systematic screens of a Candida albicans homozygous deletion library decouple morphogenetic switching and pathogenicity. Nat. Genet. 42, 590-598 (2010).

9. Ryan, O. et al. Global gene deletion analysis exploring yeast filamentous growth. Science 337, 1353-1356 (2012).

10. O'Meara, T. R. et al. Global analysis of fungal morphology exposes mechanisms of host cell escape. Nat. Commun. 6, 6741 (2015).

11. Liu, H., Kohler, J. \& Fink, G. R. Suppression of hyphal formation in Candida albicans by mutation of a STE12 homolog. Science 266, 1723-1726 (1994).

12. Leberer, E. et al. Signal transduction through homologs of the Ste20p and Ste7 protein kinases can trigger hyphal formation in the pathogenic fungus Candida albicans. Proc. Natl. Acad. Sci. USA 93, 13217-13222 (1996). 
13. Skrzypek, M. S. et al. The Candida Genome Database (CGD): incorporation of Assembly 22, systematic identifiers and visualization of high throughput sequencing data. Nucleic Acids Res. 45, D592-D596 (2017).

14. Hickman, M. A. et al. The 'obligate diploid' Candida albicans forms matingcompetent haploids. Nature 494, 55-59 (2013).

15. Shapiro, R. S. et al. A CRISPR-Cas9-based gene drive platform for genetic interaction analysis in Candida albicans. Nat. Microbiol. 3, 73-82 (2018).

16. Cary, L. C. et al. Transposon mutagenesis of baculoviruses: analysis of Trichoplusia $n i$ transposon IFP2 insertions within the FP-locus of nuclear polyhedrosis viruses. Virology 172, 156-169 (1989).

17. Ding, S. et al. Efficient transposition of the piggyBac (PB) transposon in mammalian cells and mice. Cell 122, 473-483 (2005).

18. Mitra, R., Fain-Thornton, J. \& Craig, N. L. piggyBac can bypass DNA synthesis during cut and paste transposition. EMBO J. 27, 1097-1109 (2008).

19. Elick, T. A., Bauser, C. A. \& Fraser, M. J. Excision of the piggyBac transposable element in vitro is a precise event that is enhanced by the expression of its encoded transposase. Genetica 98, 33-41 (1996).

20. Li, J. et al. A piggyBac transposon-based mutagenesis system for the fission yeast Schizosaccharomyces pombe. Nucleic Acids Res. 39, e40 (2011).

21. Yusa, K., Zhou, L., Li, M. A., Bradley, A. \& Craig, N. L. A hyperactive piggyBac transposase for mammalian applications. Proc. Natl. Acad. Sci. USA 108 1531-1536 (2011).

22. Zeng, G., Wang, Y. M., Chan, F. Y. \& Wang, Y. One-step targeted gene deletion in Candida albicans haploids. Nat. Protoc. 9, 464-473 (2014).

23. Engels, W. R., Johnson-Schlitz, D. M., Eggleston, W. B. \& Sved, J. Highfrequency $\mathrm{P}$ element loss in Drosophila is homolog dependent. Cell 62, 515-525 (1990).

24. Giaever, G. et al. Functional profiling of the Saccharomyces cerevisiae genome. Nature 418, 387-391 (2002).

25. Boeke, J. D., LaCroute, F. \& Fink, G. R. A positive selection for mutants lacking orotidine- $5^{\prime}$-phosphate decarboxylase activity in yeast: 5 -fluoro-orotic acid resistance. Mol. Gen. Genet. 197, 345-346 (1984).

26. Jones, E. W. \& Fink, G. R. Regulation of amino acid and nucleotide biosynthesis in yeast. In The Molecular Biology of the Yeast Saccharomyces: Metabolism and Gene Expression (eds Strathern, J. N. et al.) 181-299 (Cold Spring Harbor Laboratory Press, Cold Spring Harbor, NY, 1982).

27. David, D., Sundarababu, S. \& Gerst, J. E. Involvement of long chain fatty acid elongation in the trafficking of secretory vesicles in yeast. J. Cell Biol. 143, 1167-1182 (1998).

28. Oh, C. S., Toke, D. A., Mandala, S. \& Martin, C. E. ELO2 and ELO3, homologues of the Saccharomyces cerevisiae ELO1 gene, function in fatty acid elongation and are required for sphingolipid formation. J. Biol. Chem. 272, 17376-17384 (1997).

29. Kelly, S. L. et al. Resistance to fluconazole and cross-resistance to amphotericin B in Candida albicans from AIDS patients caused by defective sterol delta5,6-desaturation. FEBS Lett. 400, 80-82 (1997).

30. Bittman, R., Kasireddy, C. R., Mattjus, P. \& Slotte, J. P. Interaction of cholesterol with sphingomyelin in monolayers and vesicles. Biochemistry 33, 11776-11781 (1994)

31. Silver, P. M., Oliver, B. G. \& White, T. C. Role of Candida albicans transcription factor Upc2p in drug resistance and sterol metabolism. Eukaryot. Cell 3, 1391-1397 (2004).

32. Hallstrom, T. C. et al. Coordinate control of sphingolipid biosynthesis and multidrug resistance in Saccharomyces cerevisiae. J. Biol. Chem. 276, 23674-23680 (2001).

33. Kihara, A. \& Igarashi, Y. Cross talk between sphingolipids and glycerophospholipids in the establishment of plasma membrane asymmetry. Mol. Biol. Cell 15, 4949-4959 (2004).

34. Kolaczkowski, M., Kolaczkowska, A., Gaigg, B., Schneiter, R. \& Moye-Rowley, W. S. Differential regulation of ceramide synthase components LAC1 and LAG1 in Saccharomyces cerevisiae. Eukaryot. Cell 3, 880-892 (2004).

35. Panwar, S. L. \& Moye-Rowley, W. S. Long chain base tolerance in Saccharomyces cerevisiae is induced by retrograde signals from the mitochondria. J. Biol. Chem. 281, 6376-6384 (2006).

36. Uhl, M. A., Biery, M., Craig, N. \& Johnson, A. D. Haploinsufficiency-based large-scale forward genetic analysis of filamentous growth in the diploid human fungal pathogen C. albicans. EMBO J. 22, 2668-2678 (2003).

37. Oh, J. et al. Gene annotation and drug target discovery in Candida albicans with a tagged transposon mutant collection. PLoS Pathog. 6, e1001140 (2010).

38. Davis, D. A., Bruno, V. M., Loza, L., Filler, S. G. \& Mitchell, A. P. Candida albicans $\mathrm{Mds} 3 \mathrm{p}$, a conserved regulator of $\mathrm{pH}$ responses and virulence identified through insertional mutagenesis. Genetics 162, 1573-1581 (2002)

39. Epp, E. et al. Forward genetics in Candida albicans that reveals the Arp2/3 complex is required for hyphal formation, but not endocytosis. Mol. Microbiol. 75, 1182-1198 (2010).

40. Glazier, V. E. et al. Systematic complex haploinsufficiency-based genetic analysis of Candida albicans transcription factors: tools and applications to virulence-associated phenotypes. G3 (Bethesda) 8, 1299-1314 (2018).
41. Glazier, V. E. et al. Genetic analysis of the Candida albicans biofilm transcription factor network using simple and complex haploinsufficiency. PLoS Genet. 13, e1006948 (2017).

42. Mielich, K. et al. Maize transposable elements Ac/Ds as insertion mutagenesis tools in Candida albicans. G3 (Bethesda) 8, 1139-1145 (2018).

43. Robbins, N., Wright, G. D. \& Cowen, L. E. Antifungal drugs: the current armamentarium and development of new agents. Microbiol. Spectr. 4, https:// doi.org/10.1128/microbiolspec.FUNK-0002-2016 (2016).

44. Robbins, N., Caplan, T. \& Cowen, L. E. Molecular evolution of antifungal drug resistance. Annu. Rev. Microbiol. 71, 753-775 (2017).

45. Jensen-Pergakes, K. L. et al. Sequencing, disruption, and characterization of the Candida albicans sterol methyltransferase (ERG6) gene: drug susceptibility studies in erg6 mutants. Antimicrob. Agents Chemother. 42, 1160-1167 (1998)

46. Chau, A. S. et al. Inactivation of sterol Delta5,6-desaturase attenuates virulence in Candida albicans. Antimicrob. Agents Chemother. 49, 3646-3651 (2005).

47. Morio, F., Pagniez, F., Lacroix, C., Miegeville, M. \& Le Pape, P. Amino acid substitutions in the Candida albicans sterol Delta5,6-desaturase (Erg3p) confer azole resistance: characterization of two novel mutants with impaired virulence. J. Antimicrob. Chemother. 67, 2131-2138 (2012).

48. Luna-Tapia, A. et al. Loss of Upc2p-inducible ERG3 transcription is sufficient to confer niche-specific azole resistance without compromising Candida albicans pathogenicity. MBio 9, e00225-18 (2018).

49. Gallo-Ebert, C. et al. Arv1 lipid transporter function is conserved between pathogenic and nonpathogenic fungi. Fungal Genet. Biol. 49, 101-113 (2012).

50. Alfatah, M., Bari, V. K., Nahar, A. S., Bijlani, S. \& Ganesan, K. Critical role for CaFEN1 and CaFEN12 of Candida albicans in cell wall integrity and biofilm formation. Sci. Rep. 7, 40281 (2017).

51. Gronberg, L., Ruan, Z. S., Bittman, R. \& Slotte, J. P. Interaction of cholesterol with synthetic sphingomyelin derivatives in mixed monolayers. Biochemistry 30, 10746-10754 (1991).

52. Guan, X. L. et al. Functional interactions between sphingolipids and sterols in biological membranes regulating cell physiology. Mol. Biol. Cell 20, 2083-2095 (2009).

53. Kobayashi, S. D. \& Nagiec, M. M. Ceramide/long-chain base phosphate rheostat in Saccharomyces cerevisiae: regulation of ceramide synthesis by Elo3p and Cka2p. Eukaryot. Cell 2, 284-294 (2003).

54. Prasad, T. et al. Functional analysis of CaIPT1, a sphingolipid biosynthetic gene involved in multidrug resistance and morphogenesis of Candida albicans. Antimicrob. Agents Chemother. 49, 3442-3452 (2005).

55. Mukhopadhyay, K. et al. Membrane sphingolipid-ergosterol interactions are important determinants of multidrug resistance in Candida albicans. Antimicrob. Agents Chemother. 48, 1778-1787 (2004).

56. Singh, A. \& Del Poeta, M. Sphingolipidomics: an important mechanistic tool for studying fungal pathogens. Front. Microbiol. 7, 501 (2016).

57. Worgall, T. S. Sphingolipid synthetic pathways are major regulators of lipid homeostasis. Adv. Exp. Med. Biol. 721, 139-148 (2011).

58. Simons, K. \& Ikonen, E. Functional rafts in cell membranes. Nature 387, 569-572 (1997).

59. Lingwood, D. \& Simons, K. Lipid rafts as a membrane-organizing principle. Science 327, 46-50 (2010).

60. Lupetti, A., Danesi, R., Campa, M., Del Tacca, M. \& Kelly, S. Molecular basis of resistance to azole antifungals. Trends Mol. Med. 8, 76-81 (2002).

61. Abe, F., Usui, K. \& Hiraki, T. Fluconazole modulates membrane rigidity, heterogeneity, and water penetration into the plasma membrane in Saccharomyces cerevisiae. Biochemistry 48, 8494-8504 (2009).

62. Sharma, S. et al. Sphingolipid biosynthetic pathway genes FEN1 and SUR4 modulate amphotericin B resistance. Antimicrob. Agents Chemother. 58, 2409-2414 (2014)

63. de Kruijff, B. \& Demel, R. A. Polyene antibiotic-sterol interactions in membranes of Acholeplasma laidlawii cells and lecithin liposomes. 3. Molecular structure of the polyene antibiotic-cholesterol complexes. Biochim. Biophys. Acta 339, 57-70 (1974).

64. Andreoli, T. E. The structure and function of amphotericin B-cholesterol pores in lipid bilayer membranes. Ann. N. Y. Acad. Sci. 235, 448-468 (1974).

65. Park, Y. N. \& Morschhauser, J. Tetracycline-inducible gene expression and gene deletion in Candida albicans. Eukaryot. Cell 4, 1328-1342 (2005).

66. Livak, K. J. \& Schmittgen, T. D. Analysis of relative gene expression data using real-time quantitative PCR and the 2(-Delta Delta C(T)) Method. Methods 25, 402-408 (2001).

67. Rex, J. H. et al. Clinical and Laboratory Standards Institute (CLSI). Reference Method for Broth Dilution Antifungal Susceptibility Testing of Yeasts, Approved Standard-Third Edition, CLSI document M27-A3 (ISBN 1-56238666-2) (Clinical and Laboratory Standards Institute, 940 West Valley Road, Suite 1400, Wayne, Pennsylvania 19087-1898, USA, 2008).

68. Yuan, M., Breitkopf, S. B., Yang, X. \& Asara, J. M. A positive/negative ionswitching, targeted mass spectrometry-based metabolomics platform for bodily fluids, cells, and fresh and fixed tissue. Nat. Protoc. 7, 872-881 (2012). 
69. Bligh, E. G. \& Dyer, W. J. A rapid method of total lipid extraction and purification. Can. J. Biochem. Physiol. 37, 911-917 (1959).

\section{Acknowledgements}

We thank Dr. Yang Chen from Dr. Li Yu's lab and Dr. Feng Chen for technical assis tance. We are grateful for the support of Metabolomics Facility in Technology Center for Protein Sciences and the advice of members of J.W.'s group and Yanyi Huang's group at various stages of this work. J.G. thanks Jing Li for her support. This work was supported by the Thousand Young Talents Program (J.W.), Ministry of Science and Technology of China (2016YFC0900103 to J.W.), National Natural Science Foundation of China (21675098 to J.W.), THU-PKU Center for Life Sciences (J.G. and J.W.), and the Agency for Sciences, Technology and Research of Singapore (BMRC/BnB/0001b/2012 to Y.W.). Funding includes NIH GM117111 to H.L. We are grateful to Dr. Li-lin Du of NIBS for providing $\mathrm{pPB}[\mathrm{ura} 4]$ and $\mathrm{pDUAL}-\mathrm{PBase}$ and Allan Bradley of Sanger for hyPBase.

\section{Author contributions}

J.G., J.W., and Y.W. conceptualized, designed, and supervised the project; H.L. conceptualized, designed and supervised the project and acquired funding; J.G. conducted experiments; H.W. and Z.L. contributed to the computational analysis; A.H.-H.W., Y.-Z. W., Y.G., and X.L. provided technical assistance; G.Z. generated haploid strains; J.G., H. W., J.W., and Y.W. analyzed data and wrote the paper; J.W. and Y.W. acquired funding.

\section{Additional information}

Supplementary Information accompanies this paper at https://doi.org/10.1038/s41467018-06944-1.
Competing interests: The authors declare no competing interests.

Reprints and permission information is available online at http://npg.nature.com/ reprintsandpermissions/

Publisher's note: Springer Nature remains neutral with regard to jurisdictional claims in published maps and institutional affiliations.

\section{(c) (i)}

Open Access This article is licensed under a Creative Commons Attribution 4.0 International License, which permits use, sharing, adaptation, distribution and reproduction in any medium or format, as long as you give appropriate credit to the original author(s) and the source, provide a link to the Creative Commons license, and indicate if changes were made. The images or other third party material in this article are included in the article's Creative Commons license, unless indicated otherwise in a credit line to the material. If material is not included in the article's Creative Commons license and your intended use is not permitted by statutory regulation or exceeds the permitted use, you will need to obtain permission directly from the copyright holder. To view a copy of this license, visit http://creativecommons.org/ licenses/by/4.0/.

(C) The Author(s) 2018 